\title{
A Sublinear-Scaling Approach to Density-Functional-Theory Analysis of Crystal Defects
}

\author{
M. Ponga*, K. Bhattacharya ${ }^{\dagger}$ and M. Ortiz ${ }^{\dagger}$ \\ * Department of Mechanical Engineering, University of British Columbia, \\ 2054 - 6250 Applied Science Lane, Vancouver, BC, V6T 1Z4, Canada. \\ $\dagger$ Division of Engineering and Applied Sciences, California Institute of Technology, \\ 1200 E. California Blvd, Pasadena, CA, 91125, USA.
}

\begin{abstract}
We develop a sublinear-scaling method, referred to as MacroDFT, for the study of crystal defects using ab-initio Density Functional Theory (DFT). The sublinear scaling is achieved using a combination of the Linear Scaling Spectral Gauss Quadrature method (LSSGQ) and a CoarseGraining approach (CG) based on the quasi-continuum method. LSSGQ reformulates DFT and evaluates the electron density without computing individual orbitals. This direct evaluation is possible by recourse to Gaussian quadrature over the spectrum of the linearized Hamiltonian operator. Furthermore, the nodes and weights of the quadrature can be computed independently for each point in the domain. This property is exploited in CG, where fields of interest are computed at selected nodes and interpolated elsewhere. In this paper, we present the MacroDFT method, its parallel implementation and an assessment of convergence and performance by means of test cases concerned with point defects in magnesium.
\end{abstract}

\section{Introduction}

Density Functional Theory (DFT) of Hohenberg and Kohn [1] and Kohn and Sham [2] has emerged as the method of choice in studying the properties of matter, including the mechanical properties of solids, from first principle with little empirical input. A major drawback of the commonly used Kohn and Sham approach is that it requires the calculation of individual orbitals [3, 4, 5, 6, 7, 8, 9, subject to an orthogonality condition. The orthogonalization of the orbitals is computationally costly and renders calculations of large representative material samples prohibitively expensive. Furthermore, most parallel DFT implementations require all-to-all communication, in turn resulting in poor performance on massively parallel platforms. Finally, orbitals may be delocalized, and this hampers the effective coarse-graining of traditional DFT formulations and limits their applicability to small samples.

A number of linear scaling DFT formulations have been proposed in response to these challenges [10, 11, 12, 13, 14, 15, 16. Many of these formulations posit an exponential decay of the non-diagonal components of the density matrix. However, a detailed mathematical and 
physical understanding of the exponential decay properties of the density matrix - especially in conductors - remains unavailable and, therefore, the development of linear scaling methods for metallic systems has long been considered an open problem [17].

An additional challenge is encountered in the study of crystal defects. Defects determine critical properties of crystalline materials despite occurring at relatively low concentrations. Defects intimately couple phenomena at multiple scales, including the chemistry and atomic structure of the core and the attendant long-range elastic fields. In particular, the slow decay of the elastic fields of defects necessitates the use of exceedingly large unit cells in order to achieve convergence with respect to cell size [18, 19]. The need to simultaneously resolve the electronic structure and the long-range elastic fields of defects poses a severe technical challenge. A number of multiscale approaches have been proposed that consist of patching together heterogeneous models at different scales, from DFT to continuum elasticity. The coupling between the various models varies from simple parameter passing to hybrid Hamiltonians (e. g., [20, 21, 22, 23, 24, 25]). Since these methods bring together distinct models that embody different physics and differing mathematical formulations, they typically assume separation of scales (as in parameter passing) or additional physics or constraints at the interface (as in hybrid Hamiltonians). Often, the models need to be adjusted or calibrated to the particular phenomenon under consideration, which ultimately detracts from the predictiveness and fundamental nature of first-principles calculations.

The present work differs from the 'patched'-model approach crucially in that we strive to formulate a computational scheme scalable to large representative material samples with DFT as its sole input. In particular, in the resulting scheme, coarse-graining is strictly the result of approximation theory and, consequently, is ansatz-free and does not introduce spurious physics or uncontrolled assumptions beyond DFT. A computational framework possessing these desirable properties can be achieved by combining a local linear-scaling scheme and a seamless coarse-graining scheme. Following on a suggestion of [19, 26], in this work we specifically combine the Linear Scaling Spectral Gauss Quadrature (LSSGQ) with QuasiContinuum (QC) coarse-graining. For ease of reference, we term the resulting approach MacroDFT. The purpose of the present work is to present a systematic and comprehensive verification and validation of MacroDFT as well as an assessment of scaling and performance of the method on massively parallel platforms.

We recall [19] that the LSSGQ scheme eschews the computation of orbitals, which owing to their delocalized nature impede subsequent coarse-graining, by reinterpreting sums over occupied states as Riemann-Stieltjes integrals over the the spectrum of the linearized Hamiltonian, within a self-consistent iteration framework, and by evaluating the resulting integrals by means of Gaussian quadrature. Given the Fermi level, electron density and electrostatic potential, the spectral nodes and weights are computed using the Lanczos algorithm. The resulting LSSGQ scheme has $\mathcal{O}(N)$ complexity and excellent parallel scalability [19].

Following [18], we additionally employ two different representations for atoms and electronic fields for purposes of coarse-graining. The representation for the atoms is based on the Quasi-Continuum (QC) approach of Tadmor et al. [27] and others, consisting of the selection of a reduced set of atoms, or representative atoms, and subsequent interpolation of the positions of non-representative atoms. The coarse-graining framework for the electronic fields builds on ideas introduced by Gavini et al. [18] in the context of orbital-free density functional theory. Specifically, MacroDFT decomposes the electronic fields, such as 
partial band energy, electron density, electrostatic potential and exchange and correlation, and others, into predictor and corrector fields. The predictor fields are computed through an inexpensive periodic calculation for the perfect lattice, with the corrector fields defined as the difference between the solution and the predictor. Because the electronic perturbations tend to decay smoothly and rapidly, the corrector fields are concentrated in the vicinity of the core of the defect. Exploiting this decay property, MacroDFT computes the corrector fields at selected points, or electronic sampling points (ESPs), of a finite-difference grid and interpolates them to the remaining points on the grid. The overall computational complexity of the calculation can then be greatly reduced at no significant loss of accuracy, effectively achieving sublinear scaling, by adaptively choosing ESPs to be dense near the core and sparse elsewhere.

We validate MacroDFT by means of test cases concerned with points defects in magnesium $(\mathrm{Mg})$. Point defects supply an ideal test case for coarse-grained DFT because they are present only in dilute concentrations and exhibit a long-range elastic (dipole-like) interactions. Point defects are of interest in $\mathrm{Mg}$ and its alloys for the role they play in dynamic failure, including spallation [28], when large tensile stresses are induced in the material. A systematic study of these point defects is an ancillary goal of the present work. A key insight revealed by this study is the significant role played of Friedel-type oscillations that extend over many atomic spacings. The implementation of MacroDFT presented in this work supports MPI communication in conjunction with multi-threading and requires minimal communication between processors. The load is distributed between the different computational nodes using a custom dynamic load balance algorithm. Another ancillary of the present work is to assess, with the aid of the validation tests just described, the performance and scaling of MacroDFT on the MIRA IBM BG/Q 1.6 GHz PowerPC A2 supercomputer of Argonne National Laboratory.

The remainder of this paper is organized as follows. After some prolegomena, the theoretical framework LSSGQ is described in Section 3, and the coarse-graining methodology is described in Section 4. We subsequently discuss matters of parallel implementation of MacroDFT using HCP-Mg as a motivational example. Section 5 concerns the implementation at full resolution, i. e., no coarse-graining. We verify the accuracy of MacroDFT against prior DFT calculations, demonstrate linear scaling for up to 1,024 atoms and assess parallel performance up to 4,096 processors. In Section 6, we turn to coarse-graining. We demonstrate the accuracy and scaling of the approach, as well as the need for large computational cells even for simple defects such as vacancies. Finally, we demonstrate a parallel efficiency of nearly $75 \%$ up to 8,192 processors. We study divacancies in Section 7 and vacancies under hydrostatic pressure in Section 8. We conclude in Section 9.

\section{Density-functional theory}

Given an atomic system with an any collection of nuclei with specified position and charge, Density Functional Theory (DFT) provides the ground state energy of a system of electrons [1, 2]. We start with a brief review (ignoring spin for convenience); the reader is referred to [29, 30] for details. We specifically consider a system of $M$ atoms with $N_{e}$ electrons and let $\boldsymbol{R}=\left\{\boldsymbol{R}_{1}, \boldsymbol{R}_{2}, \ldots, \boldsymbol{R}_{M}\right\}$ denote the position of nuclei with charges $\left\{Z_{1}, Z_{2}, \ldots, Z_{M}\right\}$ respec- 
tively. Here and subsequently we assume that $N_{e}$ is an even number. The corresponding DFT energy is

$$
\begin{gathered}
\mathcal{E}(\psi, \boldsymbol{R})=T_{s}(\psi)+E_{x c}(\rho)+E_{H}(\rho)+E_{\mathrm{ext}}(\rho, \boldsymbol{R})+E_{Z Z}(\boldsymbol{R}), \\
T_{s}(\psi)=-\sum_{n=1}^{N_{e} / 2} \int_{\mathbb{R}^{3}}\left|\nabla \psi_{n}(\boldsymbol{x})\right|^{2} d x
\end{gathered}
$$

is the kinetic energy of the electrons, $E_{x c}$ is the exchange-correlation energy in the Local Density Approximation (LDA), and

$$
\begin{gathered}
E_{H}(\rho)=\frac{1}{2} \int_{\mathbb{R}^{3}} \int_{\mathbb{R}^{3}} \frac{\rho(\boldsymbol{x}) \rho\left(\boldsymbol{x}^{\prime}\right)}{\left|\boldsymbol{x}-\boldsymbol{x}^{\prime}\right|} d x d x^{\prime}, \\
E_{\text {ext }}(\rho, \boldsymbol{R})=\int_{\mathbb{R}^{3}} \rho(\boldsymbol{x})\left(\sum_{J=1}^{M} \frac{Z_{J}}{\left|\boldsymbol{x}-\boldsymbol{R}_{j}\right|}\right) d x
\end{gathered}
$$

and

$$
E_{Z Z}(\boldsymbol{R})=\frac{1}{2} \sum_{I=1}^{M} \sum_{\substack{J=1 \\ I \neq J}}^{M} \frac{Z_{I} Z_{J}}{\left|\boldsymbol{R}_{I}-\boldsymbol{R}_{J}\right|}
$$

are the Coulombic electron-electron, electron-nuclei and nuclei-nuclei interaction energies, respectively, with

$$
\rho(\boldsymbol{x})=2 \sum_{n=1}^{N_{e} / 2}\left|\psi_{n}(\boldsymbol{x})\right|^{2} .
$$

In this work, we use the LDA for the exchange-correlation:

$$
\begin{aligned}
E_{x c}(\rho(\mathbf{x})) & =E_{x}(\rho(\mathbf{x}))+E_{c}(\rho(\mathbf{x})) \text { where } \\
E_{x}(\rho(\mathbf{x})) & =-\frac{3}{4}\left(\frac{3}{\pi}\right)^{1 / 3} \int_{\mathbb{R}^{3}} \rho(\mathbf{x})^{4 / 3} d \mathbf{x}, \\
E_{c}(\rho(\mathbf{x})) & =\int_{\mathbb{R}^{3}} \varepsilon_{c}(\rho(\mathbf{x})) \rho(\mathbf{x}) d \mathbf{x}
\end{aligned}
$$

where we take $\varepsilon_{c}$ to be the parametrization proposed by Perdew and Wang [31] fitted to accurate Monte Carlo simulations carried out by Ceperley and Alder [32.

The cumbersome Coulombic double sums may be sidestepped by introducing the electrostatic potential $\phi$ as the solution to the Poisson equation [6, 33]

$$
-\frac{1}{4 \pi} \nabla^{2} \phi(\boldsymbol{x}, \boldsymbol{R})=\rho(\boldsymbol{x})+b(\boldsymbol{x}, \boldsymbol{R})
$$

where $b(\boldsymbol{x}, \boldsymbol{R})=\sum_{J=1}^{M} b_{J}\left(\boldsymbol{x}, \boldsymbol{R}_{J}\right)$ denotes the total charge density of the nuclei, with $b_{J}\left(\boldsymbol{x}, \boldsymbol{R}_{J}\right)$ representing the regularized charge density of the $J^{\text {th }}$ nucleus. Then, the electrostatic energy follows conveniently as

$$
\begin{aligned}
E_{\mathrm{H}}+E_{\mathrm{ext}}+E_{\mathrm{zz}}= & -\frac{1}{8 \pi} \int_{\mathbb{R}^{3}}|\nabla \phi(\boldsymbol{x}, \boldsymbol{R})|^{2} d x+\int_{\mathbb{R}^{3}}(\rho(\boldsymbol{x})+b(\boldsymbol{x}, \boldsymbol{R})) \phi(\boldsymbol{x}, \boldsymbol{R}) d x \\
& -\frac{1}{2} \sum_{J=1}^{M} \int_{\mathbb{R}^{3}} \int_{\mathbb{R}^{3}} \frac{b_{J}\left(\boldsymbol{x}, \boldsymbol{R}_{J}\right) b_{J}\left(\boldsymbol{x}^{\prime}, \boldsymbol{R}_{J}\right)}{\left|\boldsymbol{x}-\boldsymbol{x}^{\prime}\right|} d x d x^{\prime} .
\end{aligned}
$$


where the last term denotes the self energy of the nuclei (and is an inessential constant).

We specifically seek to determine the ground state energy of the system defined as the solution of the minimum problem

$$
\mathcal{E}_{0}=\inf _{\psi, \boldsymbol{R}} \mathcal{E}(\psi, \boldsymbol{R})
$$

subject to the orthonormality constraint

$$
\int_{\mathbb{R}^{3}} \psi_{i}^{*}(\boldsymbol{x}) \psi_{j}(\boldsymbol{x}) d x=\delta_{i j}, \quad i, j=1,2, \ldots, N_{e} / 2
$$

The conventional approach to solving this problem is to enforce the orthonormality constraint through Lagrange multipliers, which results in the nonlinear eigenvalue problem [30]

$$
\mathcal{H} \psi_{n}=\lambda_{n} \psi_{n} \quad n=1,2, \ldots N_{e} / 2
$$

where the Hamiltonian

$$
\mathcal{H}=-\frac{1}{2} \nabla^{2}+V_{x c}(\rho)+\phi(\boldsymbol{x}, \boldsymbol{R})
$$

is a self-adjoint operator over a Hilbert space $H$ with eigenvalues $\lambda_{n}$ (ordered so that $\left.\lambda_{1} \leq \lambda_{2} \leq \ldots\right)$ and $V_{x c}(\rho)=\frac{\delta E_{x c}(\rho)}{\delta \rho(\boldsymbol{x})}$. In addition, this eigenvalue problem is commonly solved by means of a fixed-point iteration known as a self-consistent field (SCF) method. In each iteration of the SCF method, the electron density is calculated by solving for the eigenfunctions $\psi_{n}$ corresponding to the lowest $N_{e} / 2$ eigenvalues $\lambda_{n}$, and then using (6). This is indeed equivalent to the variational problem (cf. e. g. [33]).

Despite its conceptual simplicity, the SCF method entails the calculation of large numbers of eigenvalues and eigenvectors - as many as the number of electrons in the system. While a variety of modifications have been developed to exploit sparsity of the matrices (e. g., iterative diagonalization in plane wave methods), these methods scale poorly, indeed as $\mathcal{O}\left(N_{e}^{3}\right)([12])$, with system size. This restricts the size of systems that can be studied. Furthermore, the orbitals have to be orthogonal and thus are are extremely oscillatory. This makes coarsegraining difficult.

\section{Linear Scaling Spectral Gauss Quadrature Method}

The Linear Scaling Spectral Gauss Quadrature (LSSGQ) method introduced in [19] effectively overcomes some of the limitations of the conventional orbital formulation of DFT described above. For completeness, we provide a brief summary of the LSSGQ method.

Observe that the energy $\mathcal{E}_{0}$ can be written as a sum over occupied states:

$$
\begin{aligned}
\mathcal{E}_{0}= & 2 \sum_{n=1}^{N_{d}} g_{0}\left(\lambda_{n}, \lambda_{f}\right) \lambda_{n}+E_{x c}(\rho)+\frac{1}{2} \int_{\mathbb{R}^{3}}(b(\boldsymbol{x}, \boldsymbol{R})-\rho(\boldsymbol{x})) \phi(\boldsymbol{x}, \boldsymbol{R}) \mathrm{d} \boldsymbol{x} \\
& -\int_{\mathbb{R}^{3}} V_{x c}(\rho) \rho(\boldsymbol{x}) \mathrm{d} \boldsymbol{x}-\frac{1}{2} \sum_{J=1}^{M} \int_{\mathbb{R}^{3}} \int_{\mathbb{R}^{3}} \frac{b_{J}\left(\boldsymbol{x}, \boldsymbol{R}_{J}\right) b_{J}\left(\boldsymbol{x}^{\prime}, \boldsymbol{R}_{J}\right)}{\left|\boldsymbol{x}-\boldsymbol{x}^{\prime}\right|} \mathrm{d} \boldsymbol{x} \mathrm{d} \boldsymbol{x}^{\prime}
\end{aligned}
$$


where $N_{d}$ are the basis functions associated to the discretization of the domain, $\lambda_{f}$ is the Fermi energy and

$$
\begin{aligned}
\rho(\boldsymbol{x}) & =\sum_{n=1}^{N_{d}} g_{0}\left(\lambda_{n}, \lambda_{f}\right)\left|\psi_{n}(\boldsymbol{x})\right|^{2} \\
g_{0}\left(\lambda_{n}, \lambda_{f}\right) & = \begin{cases}1 & \lambda_{n} \leq \lambda_{f} \\
0 & \text { else }\end{cases}
\end{aligned}
$$

where $\lambda_{n}, \psi_{n}$ are the eigenvalues and eigenvectors of the Hamiltonian (15) and $\phi$ is the solution of (10).

We first thermalize the problem to avoid discontinuous occupancy function $g_{0}$ and seek to compute the free energy

$$
\mathcal{F}=\mathcal{E}-T \mathcal{S}
$$

where

$$
\begin{aligned}
\mathcal{E}= & 2 \sum_{n=1}^{N_{d}} g\left(\lambda_{n}, \lambda_{f}\right) \lambda_{n}+E_{x c}(\rho)+\frac{1}{2} \int_{\mathbb{R}^{3}}(b(\boldsymbol{x}, \boldsymbol{R})-\rho(\boldsymbol{x})) \phi(\boldsymbol{x}, \boldsymbol{R}) \mathrm{d} \boldsymbol{x} \\
& -\int_{\mathbb{R}^{3}} V_{x c}(\rho) \rho(\boldsymbol{x}) \mathrm{d} \boldsymbol{x}-\frac{1}{2} \sum_{J=1}^{M} \int_{\mathbb{R}^{3}} \int_{\mathbb{R}^{3}} \frac{b_{J}\left(\boldsymbol{x}, \boldsymbol{R}_{J}\right) b_{J}\left(\boldsymbol{x}^{\prime}, \boldsymbol{R}_{J}\right)}{\left|\boldsymbol{x}-\boldsymbol{x}^{\prime}\right|} \mathrm{d} \boldsymbol{x} \mathrm{d} \boldsymbol{x}^{\prime} \\
= & \mathcal{E}_{\text {band }}+\mathcal{E}_{1}+\mathcal{E}_{2}+\mathcal{E}_{3}+\mathcal{E}_{\text {self }}
\end{aligned}
$$

is the energy,

$$
\mathcal{S}=-2 k_{B} \sum_{n=1}^{N_{d}}\left[g\left(\lambda_{n}, \lambda_{f}\right) \log g\left(\lambda_{n}, \lambda_{f}\right)+\left(1-g\left(\lambda_{n}, \lambda_{f}\right)\right) \log \left(1-g\left(\lambda_{n}, \lambda_{f}\right)\right)\right]
$$

is the entropy,

$$
g\left(\lambda, \lambda_{f}\right)=\frac{1}{1+\exp \left(\frac{\lambda-\lambda_{f}}{\sigma}\right)}
$$

is the Fermi-Dirac distribution with $\sigma=k_{B} T, k_{B}$ the Boltzmann constant and $T$ the absolute temperature. The Fermi energy, $\lambda_{f}$, is determined from the constraint

$$
N_{e}=2 \sum_{n=1}^{N_{d}} g\left(\lambda_{n}, \lambda_{f}\right)
$$

The reformulation of the DFT outlined so far still requires the evaluation of $N_{d}$ eigenvalues and eigenvectors. The LSSGQ method sidesteps this complete evaluation by recognizing the sum over occupied states as a Riemann-Stieltjes integral over the spectrum of the Hamiltonian (15), and approximating these spectral integrals by means of Gaussian quadrature [34]. We begin by discretizing space by means of a uniform finite-difference grid $\mathcal{T}_{\mathrm{f}}$, spanning a point set $\mathcal{P}_{\mathrm{f}}$, setting the maximal spatial resolution allowed for all electronic fields. By 
virtue of this discretization, the Hilbert space $H$ is reduced to a finite dimension $N_{\mathrm{f}}$. The Laplace operator in (15) is further approximated by means of a finite-difference scheme (cf., e. g., [19] for an analysis of convergence of finite-difference approximations). Let $\left\{\eta_{p}\right\}_{p=1}^{N_{\mathrm{f}}}$ be the orthonormal basis of $H$ associated with the uniform finite-difference grid $\mathcal{T}_{\mathrm{f}}$; then any vector $\zeta \in H$ admits the representation $\zeta=\sum_{p=1}^{N_{\mathrm{f}}} \zeta_{p} \eta_{p}$. With each basis function $\eta_{p}$ we associate a set of spectral Gauss quadrature nodes $\left\{\lambda_{k}^{\eta_{p}}\right\}$ and weights $\left\{w_{k}^{\eta_{p}}\right\}$. These quadrature nodes and weights allow us to rewrite the contributions to the free energy as

$$
\begin{aligned}
& \mathcal{E}_{\text {band }} \approx 2 \sum_{p=1}^{N_{\mathrm{f}}} \sum_{k=1}^{K} w_{k}^{\eta_{p}} \lambda_{k}^{\eta_{p}} g\left(\lambda_{k}^{\eta_{p}}, \lambda_{f}\right) \\
& \mathcal{E}_{1} \approx h^{3} \sum_{p=1}^{N_{\mathrm{f}}} E_{x c}\left(\rho_{p}\right) \\
& \mathcal{E}_{2} \approx h^{3} \sum_{p=1}^{N_{\mathrm{f}}}\left(b_{p}-\rho_{p}\right) \phi_{p} \\
& \mathcal{E}_{3} \approx-h^{3} \sum_{p=1}^{N_{\mathrm{f}}} V_{x c}\left(\rho_{p}\right) \rho_{p} \\
& S \approx-2 k_{B} \sum_{p=1}^{N_{\mathrm{f}}} \sum_{k=1}^{K} w_{k}^{\eta_{p}}\left[g\left(\lambda_{k}^{\eta_{p}}, \lambda_{f}\right) \log g\left(\lambda_{k}^{\eta_{p}}, \lambda_{f}\right)+\left(1-g\left(\lambda_{k}^{\eta_{p}}, \lambda_{f}\right)\right) \log \left(1-g\left(\lambda_{k}^{\eta_{p}}, \lambda_{f}\right)\right)\right] .
\end{aligned}
$$

where $h$ is the finite-difference spacing and $K$ is the degree of the quadrature. Further, the Fermi level and the electron density, which are itself approximated as

$$
\begin{aligned}
N_{e} & \approx 2 \sum_{p=1}^{N_{\mathrm{f}}} \sum_{k=1}^{K} w_{k}^{\eta_{p}} g\left(\lambda_{k}^{\eta_{p}}, \lambda_{f}\right), \\
\rho_{p} & \approx 2 h^{-3} \sum_{k=1}^{K} w_{k}^{\eta_{p}} g\left(\lambda_{k}^{\eta_{p}}, \lambda_{f}\right),
\end{aligned}
$$

The problem is now reduced to finding the nodes $\left\{\lambda_{k}^{\eta_{p}}\right\}$ and weights $\left\{w_{k}^{\eta_{p}}\right\}$ for the Hamiltonian $\mathcal{H}$. Conveniently, these can be computed through a Lanczos iteration that requires only matrix-vector multiplications. Critically, the vector is sparse with the number of non-zero entries dependent only on the order of the spectral quadrature and independent of system size. Thus, each evaluation is independent of the system size. This eventually gives rise to the linear scaling [19].

The calculation is iterative in nature similar to the SCF iteration. Given a Fermi level, electron density and electrostatic potential, the spectral nodes and weights are now computed from the Lanczos algorithm. The Fermi level and electron density are obtained from (27) and (28) respectively, and the electrostatic potential is recomputed from Poisson's equation (10). The iteration continues till convergence is achieved in $V_{e f f}$. The energy can now be computed from (26). 


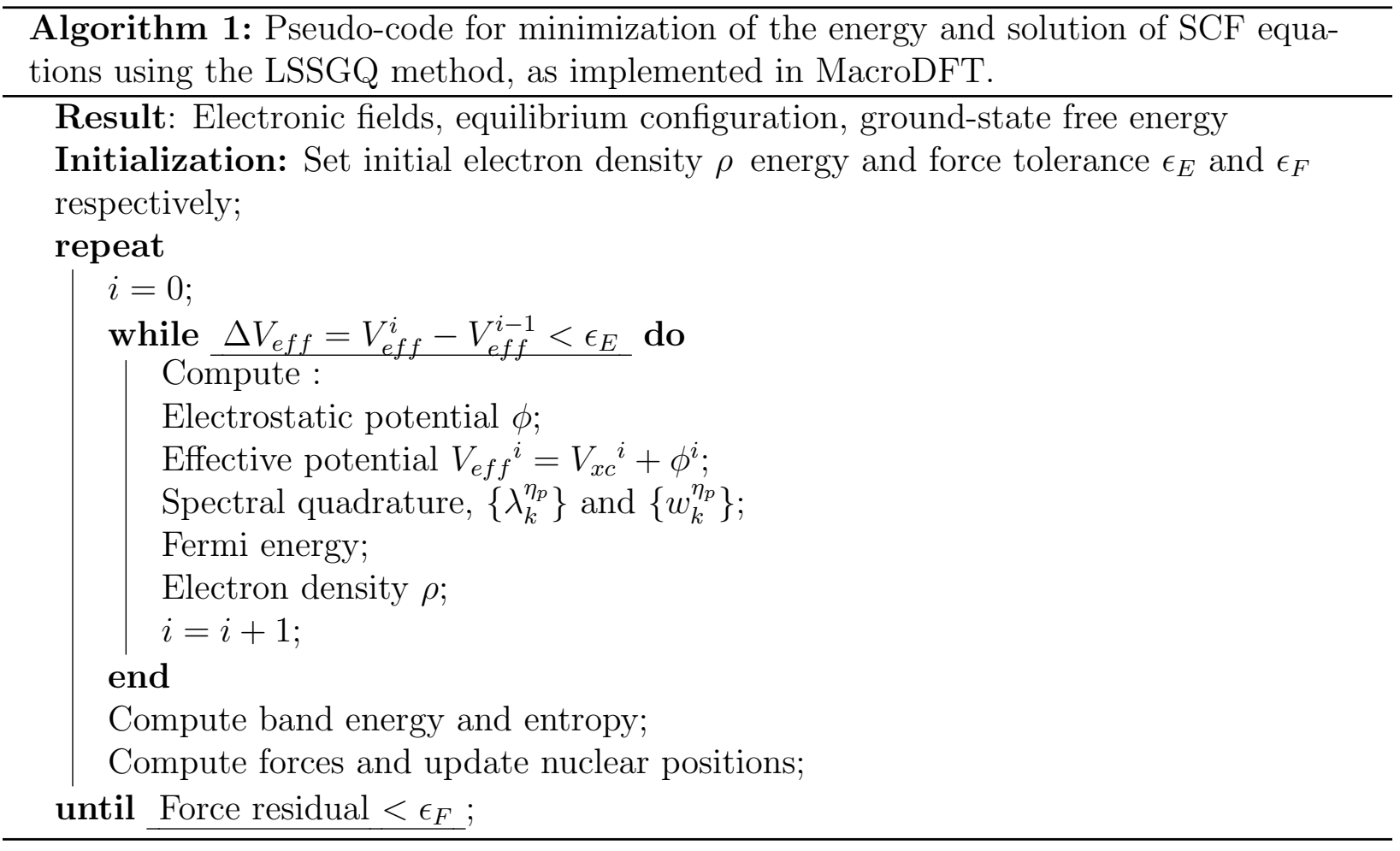

Finally, the atomic positions are relaxed by further minimizing the free energy with respect to the positions of the nuclei. This accomplished by exploiting the Hellman-Feynmann theorem [19].

The flow of calculations is summarized in Algorithm 1 .

We note that LSSGQ is related to the density matrix or operator formulation of DFT. However, the linear scaling is obtained, not by truncating the matrix, but by representing the density matrix as a spectral integral and evaluating the integral using Gaussian quadrature and Lanczos algorithm. We refer the reader to Wang et al. [35] for a complete mathematical and variational treatment of the operator formulation.

Pseudopotential approximation. It is common to use a pseudopotential approximation where the nuclear potential is replaced by a pseudopotential that accounts for the nuclei as well as core electrons, and the calculation is limited to the chemically active valence electron states. In this work, we use a local pseudopotential proposed by Huang and Carter [36] where the nuclear potential $V_{\text {ext }}$ is replaced by an explicit function $V_{\text {ext }}^{P S}(\mathbf{x}, \mathbf{R})$. This is incorporated into LSSGQ by setting $b(\mathbf{x}, \mathbf{R})=(-1 / 4 \pi) \nabla^{2} V_{e x t}^{P S}(\mathbf{x}, \mathbf{R})$ in 10$)$.

We also note that the LSSGQ formulation is consistent with the wide class of non-local pseudo-potential (e. g. [37]) that can be written in the Kleinman-Bylander form. Here, $V_{\text {ext }}$ is replaced with a self-adjoint operator that has a finite range of interaction [38. Briefly, $\phi$ in (15) is replaced with an self-adjoint operator which has a finite range of interaction, and the Hamiltonian operator retains all the properties necessary for the Gaussian quadratures. 
Properties of LSSGQ We note an important property of LSSGQ which is useful in our subsequent work. Recall $\mathcal{E}_{\text {band }}$ from $(21)$. Since the orbitals are orthonormal, $\int_{\mathbb{R}^{3}}\left|\psi_{n}\right|^{2} \mathrm{~d} \boldsymbol{x}=1$ for all $n$. Therefore,

$$
\begin{aligned}
\mathcal{E}_{\text {band }} & =2 \sum_{n=1}^{N_{d}} g\left(\lambda_{n}, \lambda_{f}\right) \lambda_{n}=2 \sum_{n=1}^{N_{d}}\left(g\left(\lambda_{n}, \lambda_{f}\right) \lambda_{n} \int_{\mathbb{R}^{3}}\left|\psi_{n}(\boldsymbol{x})\right|^{2} \mathrm{~d} \boldsymbol{x}\right) \\
& =2 \int_{\mathbb{R}^{3}}\left(\sum_{n=1}^{N_{d}} g\left(\lambda_{n}, \lambda_{f}\right) \lambda_{n}\left|\psi_{n}(\boldsymbol{x})\right|^{2}\right) \mathrm{d} \boldsymbol{x} \\
& =2 \int_{\mathbb{R}^{3}} e_{\text {band }}(\boldsymbol{x}) \mathrm{d} \boldsymbol{x} .
\end{aligned}
$$

where

$$
e_{\text {band }}(\boldsymbol{x})=\sum_{n=1}^{N_{d}} g\left(\lambda_{n}, \lambda_{f}\right) \lambda_{n}\left|\psi_{n}(\boldsymbol{x})\right|^{2} .
$$

We can similarly rewrite $\mathcal{S}$ as

$$
\mathcal{S}=\int_{\mathbb{R}^{3}} s(\boldsymbol{x}) \mathrm{d} \boldsymbol{x}
$$

where

$$
s(\boldsymbol{x})=k_{B} \sum_{n=1}^{N_{d}}\left(g\left(\lambda_{n}, \lambda_{f}\right) \log g\left(\lambda_{n}, \lambda_{f}\right)+\left(1-g\left(\lambda_{n}, \lambda_{f}\right)\right) \log \left(1-g\left(\lambda_{n}, \lambda_{f}\right)\right)\right)\left|\psi_{n}(\boldsymbol{x})\right|^{2} .
$$

Consequently, the free energy $\mathcal{F}$ in $(20)$ does not depend on all the details of the individual orbitals, but on fields $e_{\text {band }}(32), s(34), \rho(6)$ and $\phi$ (obtained from $\rho$ via $(10)$ ). The orbitals are highly oscillatory (due to exclusion or orthogonality), but the fields $e_{\text {band }}, s, \rho$ and $\phi$ that reflect the collective behavior of all orbitals are much smoother. They are still periodic in periodic systems, but smoother. This will play a critical role in our coarse-graining.

One of the key properties of LSSGQ is that it provides a means to directly compute these fields without having to explicitly compute the orbitals. Indeed, recalling any vector $\zeta \in H$ admits the representation $\zeta=\sum_{p=1}^{N_{\mathrm{f}}} \zeta_{p} \eta_{p}$, it is easy to show that

$$
e_{\text {band }}(\boldsymbol{x}) \approx \sum_{p=1}^{N_{\mathrm{f}}} e_{\text {band }}^{p} \eta_{p}(\boldsymbol{x}) \quad \text { where } \quad e_{\text {band }}^{p}=h^{-3} \sum_{k=1}^{K} w_{k}^{\eta_{p}} \lambda_{k}^{\eta_{p}} g\left(\lambda_{k}^{\eta_{p}}, \lambda_{f}\right)
$$

and analogously for $s, \rho$. Since $w_{k}^{\eta_{p}}, \lambda_{k}^{\eta_{p}}$ can be computed at each $p$ at a cost independent of system size, and since the basis is local, $e_{\mathrm{band}}^{p}, \rho^{p}, s^{p}$ can be computed at each $p$ independently at a cost that is independent of the system size.

\section{Coarse-graining}

We proceed to define a spatial coarse-graining scheme for the LSSGQ method described in the foregoing. The aim is to introduce controlled approximations reducing the overall complexity of the computational scheme without compromising accuracy or introducing additional spurious physics. 
We first explain the approach heuristically. Consider an isolated crystal defect such as a vacancy cluster. The atomic positions and the electronic fields are complex close to the defect but decay elsewhere. In particular, the atomic displacement decays according to linear elasticity in either a polynomial or logarithmic manner. We can exploit this decay and approximate the position of all atoms by tracking only a set $\mathcal{P}_{\mathrm{QC}}$ of Representative Atoms (RAs) that are fully dense near the core and increasingly sparse elsewhere - shown schematically in Figure 1 and in detail in Figure 2 - and interpolating linearly between them. A natural implementation may be based on a triangulation $\mathcal{T}_{\mathrm{QC}}$ of $\mathcal{P}_{\mathrm{QC}}$. This interpolation provides a coarse-grained representation of the atomistic fields.

Next, we turn to the electronic fields. We represent the Hamiltonian on a uniformlyfine spatial finite-difference mesh of points $\mathcal{P}_{\mathrm{f}}$ as shown. However, we do not explicitly evaluate all the electronic fields - $e_{\text {band }}, s, \rho$ and $\phi-$ at each point of this mesh. Instead, we find a representation based on the following observation. Sufficiently far away from the defects, the atomic positions are locally periodic (with periodicity related to the strain in that neighborhood) and the electronic fields $-e_{\text {band }}, s, \rho$ and $\phi-$ are also almost locally periodic. Thus, we may represent

$$
\rho(x)=\rho^{0}(x)+\rho^{c}(x)
$$

and analogously for $e_{\text {band }}, s$ and $\phi$, where $\rho^{0}(x)$ is locally periodic and $\rho^{c}(x)$ decays smoothly away from the defect. We call $\rho^{0}(x)$ the predictor and obtain it from periodic unit cell calculations in each region of atomistic interpolation. We call $\rho^{c}(x)$ the corrector and exploit its decay property to represent it on the set $\mathcal{P}_{c}$ of Electronic Sampling Points (ESPs), which is a subset of the uniformly fine finite-difference mesh $\mathcal{P}_{\mathrm{f}}$, and interpolate to the remaining points of $\mathcal{P}_{\mathrm{f}}$. The three discretizations we carry in the calculations are shown schematically in Figure 1.

We evaluate these fields as follows. Given the positions of the representative atoms, we partition the spatial domain according to the triangulation $\mathcal{T}_{\mathrm{QC}}$. In each element, we perform a periodic unit-cell calculation based on the strain at that region. This operation is inexpensive because of the small size of the periodic calculations and because the number of atomic interpolation regions is small compared to the number of atoms in the system. We then assemble the unit-cell fields together using an $L^{2} \rightarrow H^{1}$ map to obtain the piecewise predictors $\rho^{0}$ and others. Independently, using the representation of the Hamiltonian based on a uniformly-fine spatial finite-difference mesh of points $\mathcal{P}_{\mathrm{f}}$, we evaluate the electronic fields $\rho, e_{\text {band }}, s$ and $\phi$ on the ESPs. In light of the properties of LSSGQ, each evaluation is independent of the system size and thus scales as $\mathcal{O}\left(\# \mathcal{P}_{c}\right)$. We then use $(36)$ to evaluate the corrector fields $\rho^{c}$ and others at the ESPs. We extend to the fine finite-difference mesh by interpolation based on a triangulation $\mathcal{T}_{c}$ of the ESPs. We now have both the predictor and the corrector on the fine mesh and a coarse-grained representation of the electronic fields.

To be precise, we carry three discretizations, Figure 2; i) a uniform finite-difference grid $\mathcal{T}_{\mathrm{f}}$ spanning a point set $\mathcal{P}_{\mathrm{f}}$, as in the LSSGQ method previously described; ii) a triangulation $\mathcal{T}_{\mathrm{c}}$ spanning a subset $\mathcal{P}_{\mathrm{c}}$ (the set of electronic sampling points or ESPs) of the $\mathcal{P}_{\mathrm{f}}$, and iii) a second triangulation $\mathcal{T}_{\mathrm{QC}}$ spanning a subset $\mathcal{P}_{\mathrm{QC}}$ (set of representative atoms or RAs) of the atoms. We choose $\mathcal{P}_{\mathrm{c}}$ so that it coincides with $\mathcal{P}_{\mathrm{f}}$ in the vicinity of the defect, thus achieving a full sub-atomic resolution in that region. Away from the defect, $\mathcal{P}_{\mathrm{c}}$ is gradually 


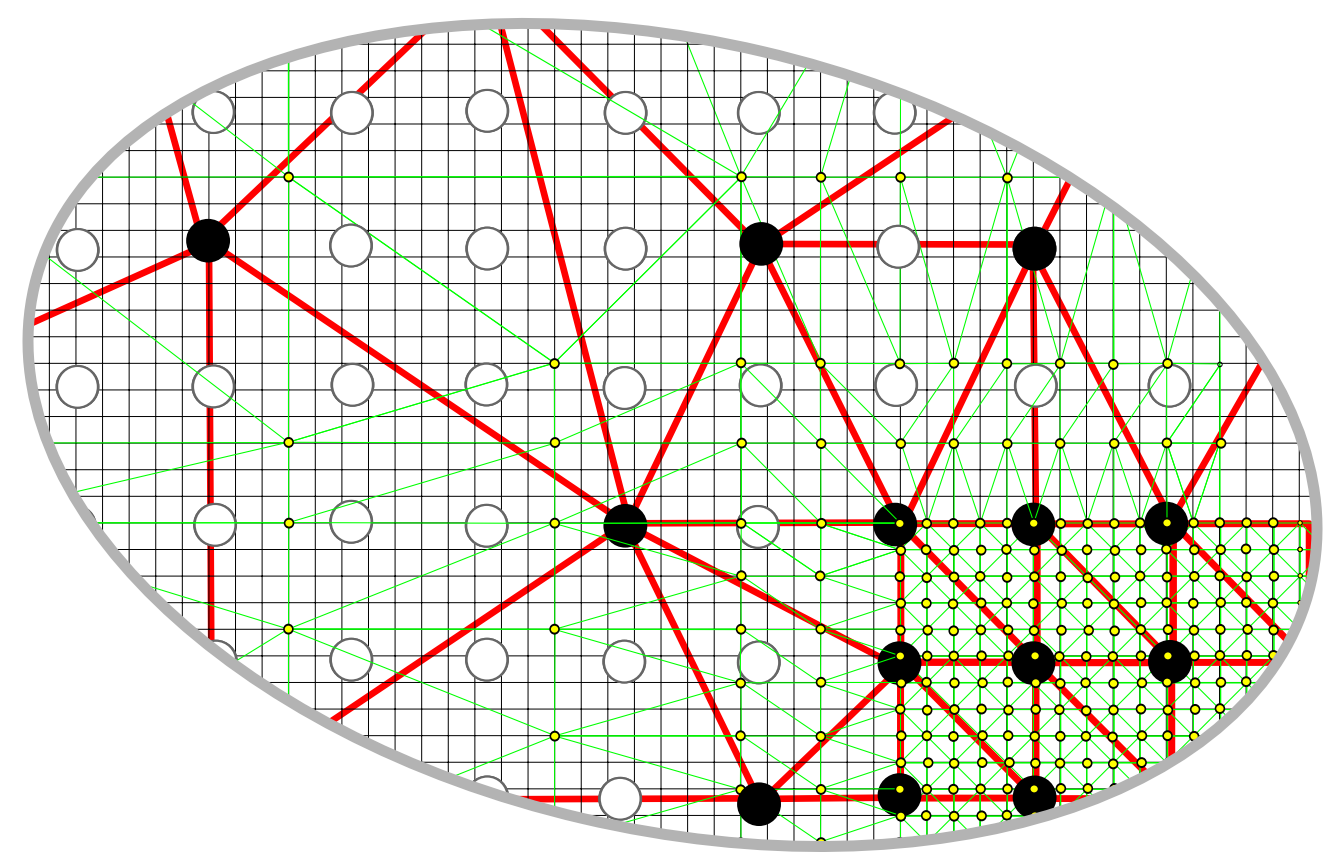

Figure 1: Schematic representation of the three discretizations used to represent the fields in a specimen where a defect is located near the bottom right. The large circles show the atoms in the current position; the filled circles show the representative atoms $\mathcal{P}_{Q C}$ and the open circles the atoms that are interpolated. Note that all atoms are representative atoms close to the defect and gradually become sparse elsewhere. The bold red lines show the finite element mesh $\mathcal{T}_{Q C}$ that spans $\mathcal{P}_{Q C}$. The thin grey lines represent the uniform fine spatial finite-difference mesh $\mathcal{P}_{f}$ which is used to represent the Hamiltonian. The small yellow circles represent the electronic sampling points $\mathcal{P}_{c}$ which is a subset of the fine mesh $\mathcal{P}_{f}$. Again, note that ESPs are dense near the defect but become increasingly sparse elsewhere.

coarse-grained. Both uniform finite difference grid $\mathcal{T}_{\mathrm{f}}$ and the triangulation $\mathcal{T}_{\mathrm{c}}$ are fixed in space, i. e., are Eulerian, whereas $\mathcal{T}_{\mathrm{QC}}$ deforms with the RAs.

We remark that the motion of atoms does not pose a problem as long as the finitedifference grid is fine enough to resolve the fields. This issue is not unique to our method, but also arises in other finite-difference based methods like PARSEC [3].

We obtain the atomistic positions by linearly interpolating the position of the nonrepresentative atoms from the representative atoms $\mathcal{P}_{\mathrm{QC}}$ :

$$
\boldsymbol{R}_{a}=\sum_{b \in \mathcal{P}_{\mathrm{QC}}} \Gamma_{a b} \boldsymbol{R}_{b}, \quad a \in \text { non-representative atoms, }
$$

where $\Gamma_{a b}$ are the weights obtained from the triangulation $\mathcal{T}_{\mathrm{QC}}$ in the reference crystal. Note that the atoms suffer affine deformation in each element of $\mathcal{T}_{\mathrm{QC}}$.

We decompose all electronic fields into predictor and corrector components, and approximate the corrector fields by interpolation on $\mathcal{T}_{\mathrm{c}}$, leading to the representations

$$
\phi_{p}=\phi_{p}^{0}+\phi_{p}^{c}, \quad \phi_{p}^{c}=\sum_{q \in \mathcal{P}_{c}} \gamma\left(\boldsymbol{r}_{p}, \boldsymbol{r}_{q}\right) \phi_{q}^{c}, \quad p \in \mathcal{P}_{\mathrm{f}},
$$




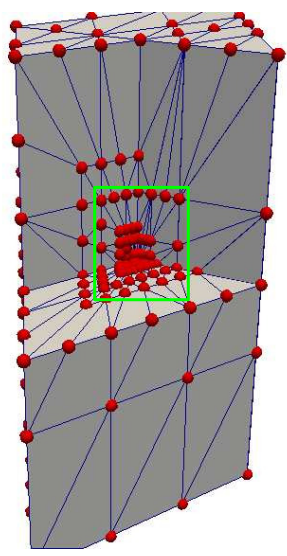

(a)

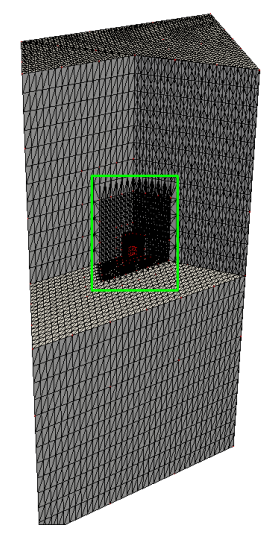

(d)

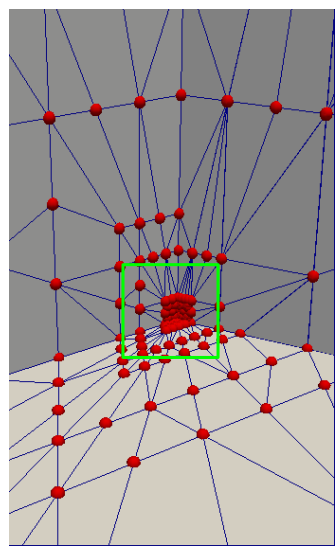

(b)

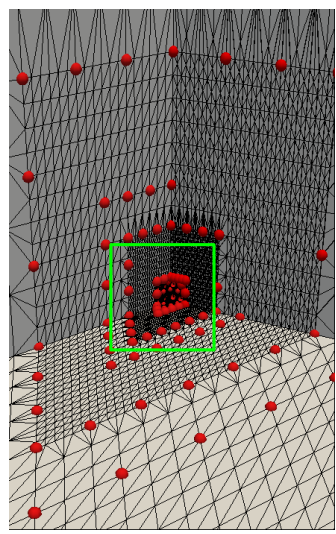

(e)

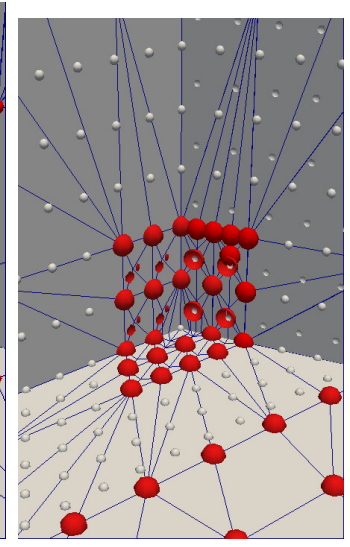

(c)

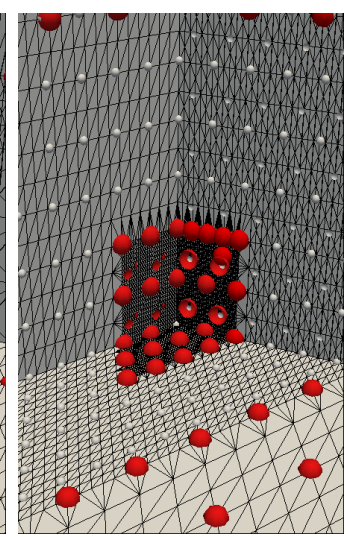

(f)

Figure 2: Coarse-graining scheme for a crystal with a defect at the center of the computational cell. Top $(\mathrm{a}, \mathrm{b}, \mathrm{c})$ : The atomic positions are obtained by interpolation using the triangulation $\mathcal{T}_{\mathrm{QC}}$ shown in blue from the set of representative atoms $\mathcal{P}_{\mathrm{QC}}$ shown in red. The figures (a)-(c) represent successive zooms: the green box in (a) and (b) indicates the region shown in detail in (b) and (c) respectively. The non-representative atoms are shown in (c) in white. Note that the set of representative atoms at $\mathcal{P}_{\mathrm{QC}}$ includes all atoms close to the core but fewer and fewer atoms away from it. Bottom (d,e,f): The corrector field is computed on set of Electronic Sampling Points or ESPs $\mathcal{P}_{\mathrm{c}}$ shown as the vertices of the triangulation $\mathcal{T}_{\mathrm{c}}$ shown in black. The figures (d)-(f) represent successive zooms: the green box in (d) and (e) indicates the region shown in detail in (e) and (f) respectively. The ESPs $\mathcal{P}_{\mathrm{c}}$ are a subset of the uniform finite-difference grid $\mathcal{P}_{\mathrm{f}}$. The two coincide close to the defect as in (f) but $\mathcal{P}_{\mathrm{c}}$ coarsens away from it. The atomic mesh $\mathcal{P}_{\mathrm{QC}}$ moves with the atoms (Lagrangean), but the electronic meshes $\mathcal{P}_{\mathrm{c}}, \mathcal{P}_{\mathrm{f}}$ are fixed in space (Eulerian). 
for the electrostatic potential, and

$$
\rho_{p}=\rho_{p}^{0}+\rho_{p}^{c}, \quad \rho_{p}^{c}=\sum_{q \in \mathcal{P}_{\mathrm{c}}} \gamma\left(\boldsymbol{r}_{p}, \boldsymbol{r}_{q}\right) \rho_{q}^{c}, \quad p \in \mathcal{P}_{\mathrm{f}},
$$

for the electron density, where $\phi_{p}^{0}$ and $\rho_{p}^{0}$ are the predictor electrostatic potential and electron density, respectively, $\phi_{q}^{c}$ and $\rho_{q}^{c}, q \in \mathcal{P}_{\mathrm{c}}$, are the nodal values of the corresponding corrector fields, and $\gamma\left(\cdot, \boldsymbol{r}_{q}\right)$ are piecewise-linear interpolation functions defined on the triangulation $\mathcal{T}_{c}$. The predictor fields are obtained by inexpensive periodic calculations on the unit cell in each element of $\mathcal{T}_{\mathrm{QC}}$, 'pasted' together by means of an $L^{2} \rightarrow H^{1}$-projection [18]. The correctors in turn account for the strong deviations from the periodic fields in the vicinity of the defect and require fine subatomic resolution in that region. By contrast, the corrector fields may be expected to decay rapidly away from the defect, thus allowing for spatial coarse-graining at no significant reduction in accuracy.

We introduce the following approximations based on representations (38) and (39).

Poisson equation and calculation of the electrostatic field. We calculate the corrector electrostatic field by a collocation method, consisting of enforcing a finite-difference approximation of Poisson's equation (10) at the ESPs in $\mathcal{P}_{\mathrm{c}}$, with the electrostatic field approximated in the corresponding stencils by means of the piecewise linear interpolation (38). Since the evaluation of the discrete Laplace operator is computed by a local finite-difference stencil the complexity of this operation is $\mathcal{O}\left(\# \mathcal{P}_{\mathrm{c}}\right)$. Further, this collocation scheme reduces exactly to LSSGQ in the fully-resolved regions.

Spectral Gaussian quadrature. We calculate the spectral Gauss quadrature nodes and weights $\left\{\lambda_{k}^{\eta_{q}}\right\}$ and $\left\{w_{k}^{\eta_{q}}\right\}$ on the ESPs $q \in \mathcal{P}_{\mathrm{c}}$ only. We note that in this calculation, the Lanczos vectors remain of bounded support around the corresponding ESPs as explained earlier, with the size of the support determined by the order of the Gauss quadrature and finite-difference stencil, but independent of the system size. This ensures that the spectral Gaussian quadrature evaluation is of $\mathcal{O}\left(\# \mathcal{P}_{\mathrm{c}}\right)$ complexity, as required.

Charge Density and Fermi energy. We represent the charge density and the charge density corrector of ESP $q \in \mathcal{P}_{c}$ as

$$
\rho_{q}=\sum_{k=1}^{K} w_{k}^{q} g\left(\lambda_{k}^{q}, \lambda_{f}\right), \quad \rho_{q}^{c}=\rho_{q}-\rho_{q}^{0}
$$

where $\rho^{0}$ is the predictor.

We now represent the number of electrons in the system as

$$
N_{e}=N_{e}^{0}+N_{e}^{c} \quad \text { where } \quad N_{e}^{0}=h^{3} \sum_{\mathcal{P}_{f}} \rho_{p}^{0} \text { and } N_{e}^{c}=h^{3} \sum_{\mathcal{P}_{f}} \rho_{p}^{c} .
$$

The first term is easily evaluated from a predictor. To evaluate the second term $N_{e}^{c}$, we recognize it to be an integral of a smooth function $\rho^{c}$ over the entire computational domain, 
and whose values are known at the $\operatorname{ESPs}_{\mathcal{c}} \mathcal{P}_{c}$. Therefore we evaluate it using Gauss-Lobatto nodal quadratures (e. g. [39]) using the ESPs as the integration point. We obtain

$$
N_{e}^{c}=\sum_{q \in \mathcal{P}_{\mathrm{c}}} n_{q} \rho_{q}^{c}
$$

where $n_{q}$ are the Lobatto weights associated to the ESPs. The use of Gauss-Lobatto nodal quadrature ensures $\mathcal{O}\left(\# \mathcal{P}_{c}\right)$ complexity. We use the number of electrons in the system as a constraint to determine the Fermi energy.

Calculation of the energy. We note from (26) that each term in the energy, and consequently the free energy, is a sum over $\mathcal{P}_{\mathrm{f}}$

$$
\mathcal{F}=\sum_{\mathcal{P}_{f}} \mathcal{F}_{p}
$$

The direct evaluations would take linear effort $\mathcal{O}\left(\# \mathcal{P}_{\mathrm{f}}\right)$. So we seek to use the coarse-grained representation to simplify the effort. However, this takes care since the energy density is non-linear in the underlying electronic fields. Consider for example, the term $\rho_{p} \phi_{p}$ (cf. 26c). Using the predictor-corrector representation 36 and 38 for $\rho_{p}, \phi_{p}$,

$$
\rho_{p} \phi_{p}=\left(\rho_{p}^{0}+\rho_{p}^{c}\right)\left(\phi_{p}^{0}+\phi_{p}^{c}\right)=\rho_{p}^{0} \phi_{p}^{0}+\left(\rho_{p}^{0} \phi_{p}^{c}+\rho_{p}^{c} \phi_{p}^{0}\right)+\rho_{p}^{c} \phi_{p}^{c} .
$$

Note that the difference $\rho_{p} \phi_{p}-\rho_{p}^{0} \phi_{p}^{0}$ between the actual value and the predictor still contains oscillatory terms (those in parenthesis). So we cannot directly use the predictor-corrector representation analogous to (36) for the energy. At the same time, we note that two problematic terms in the parenthesis are almost-periodic: periodic with a smoothly varying amplitude away from the defect. This is true of each of the terms in the energy. We exploit this property using cluster summation as in the quasi-continuum method of Knap and Ortiz [40]. The basic idea is to use the full sum (43) in the core region, but sample the energy within a cluster around each ESP in the coarse-grained region.

Set $\mathcal{C}_{q}$ to be the set or cluster of nodal points on the fine mesh contained in a ball of radius $r$ (to be decided) around the $q$ th ESP. Let $\mathcal{P}_{c}^{r}$ be the set of ESPs that are within a distance $2 r$ of another ESP. We take the union of these overlapping clusters to be one core cluster, $C_{r}=\cup_{q \in P_{c}^{r}} \mathcal{C}_{q}$. We approximate the total free energy as

$$
\mathcal{F}=\sum_{p \in \mathcal{C}_{r}} \mathcal{F}_{p}+\sum_{q \in\left(\mathcal{P}_{c} \backslash \mathcal{P}_{c}^{r}\right)} N_{q} \overline{\mathcal{F}}_{q}
$$

where $N_{q}$ is the number of points on the fine grid $\mathcal{P}_{f}$ in the Voronoi cell around the $q$ th ESP and where

$$
\overline{\mathcal{F}}_{q}=\frac{1}{N} \sum_{p \in \mathcal{C}_{q}} \mathcal{F}_{p}
$$

is the average of $\mathcal{F}_{p}$ over this cluster. We choose $r$ to be larger than the size of the crystal unit cell but small compared to the distance between ESPs in the coarse-grained regions. The effort to compute the energy (45) scales as $\mathcal{O}\left(\# \mathcal{P}_{\mathrm{c}}\right)$. Further, 45 converges to the full 
sum (43) as the resolution of ESPs increases.

Calculation of the forces on representative atoms. The force on an atom is given by the negative of the derivative of the free energy with respect to the nuclear position, and usually calculated using the Hellmann-Feynman theorem (see [29] for a general discussion and [19] for interpretation in LSSGQ). However, in our coarse-grained representation, the position of an individual atom is determined by the position of the representative atoms. Therefore, we need to compute the force on said representative atoms. We define the force on the $b$ th representative as the negative of the derivative of the free energy with respect to its nuclear position,

$$
\boldsymbol{f}_{b}=-\frac{\partial \mathcal{F}}{\partial \boldsymbol{R}_{\boldsymbol{b}}}=-\frac{\partial}{\partial \boldsymbol{R}_{\boldsymbol{b}}} \int_{\Omega} b(\boldsymbol{x}) \phi(\boldsymbol{x}) d \boldsymbol{x} .
$$

The second equality follows from the fact that $b$ is the only term that explicitly depends on atomic position and from the electronic fields being in equilibrium.

We exploit the structure of $b$ and our coarse-grained representations (37) and (36) to obtain a simple representation for this force. We present the details in the appendix under the assumption that the underlying crystal is a Bravais lattice (one atom per unit cell) and that all atoms are identica $]^{1}$. Under these assumptions, $b(\boldsymbol{x})=\sum_{a \in \mathcal{P}_{a}} b_{0}\left(\boldsymbol{x}-\boldsymbol{R}_{a}\right)$ for given $b_{0}$ that is obtained from the regularized nuclear charge (and possibly the local contribution of the pseudopotential) where the sum is taken over the set of all atoms $\mathcal{P}_{a}$. Further, $b_{0}$ has very local support. We obtain

$$
\boldsymbol{f}_{b}= \begin{cases}-\int_{\Omega} \sum_{a \in \mathcal{P}_{a}}\left(b_{0}\left(\boldsymbol{x}-\sum_{c \in \mathcal{P}_{\mathrm{QC}}} \Gamma_{a c} \boldsymbol{R}_{b}\right) \Gamma_{a b}\right) \nabla \phi(\boldsymbol{x}) d \boldsymbol{x} & b \in \mathcal{P}_{\mathrm{QC}}^{R} \\ -\sum_{e \in \mathcal{T}_{\mathrm{QC}}^{b}} \sum_{f \in \mathcal{T}_{c}^{e}} w_{e b}^{f}\left(Z \nabla \phi_{f}^{c}+\int_{\Omega_{0}^{e}} b_{0}(\boldsymbol{x}) \nabla \phi^{0}(\boldsymbol{x}) d \boldsymbol{x}\right) & b \in\left(\mathcal{P}_{\mathrm{QC}} \backslash \mathcal{P}_{\mathrm{QC}}^{R}\right)\end{cases}
$$

where $\Gamma_{a b}$ is the atomistic interpolation $(37), \mathcal{P}_{\mathrm{QC}}^{R}$ is the set of all representative atoms that is within a distance of $R$ of another representative atom, $\mathcal{T}_{\mathrm{Q} C}^{b}$ is the set of atomistic elements that contain representative atom $b$ as a node, $\mathcal{T}_{c}^{e}$ is the set of ESP elements that intersect with the atomistic element $e, w_{e b}^{f}$ is a suitable weight defined in $(60), Z=\int_{\Omega} b_{0}(\boldsymbol{x}) d \boldsymbol{x},-\nabla \phi_{f}^{c}$ is the corrector electric field in the ESP element $f$ and $\Omega_{0}^{e}$ is the crystalline unit cell in the eth atomistic element. We choose $R$ to be large compared to typical interatomic distance and small compared to the size of the elements in the coarse-grained region. Then, this force can be computed for each representative atom at $\mathcal{O}(1)$ effort.

The method is summarized in Algorithm 2. 


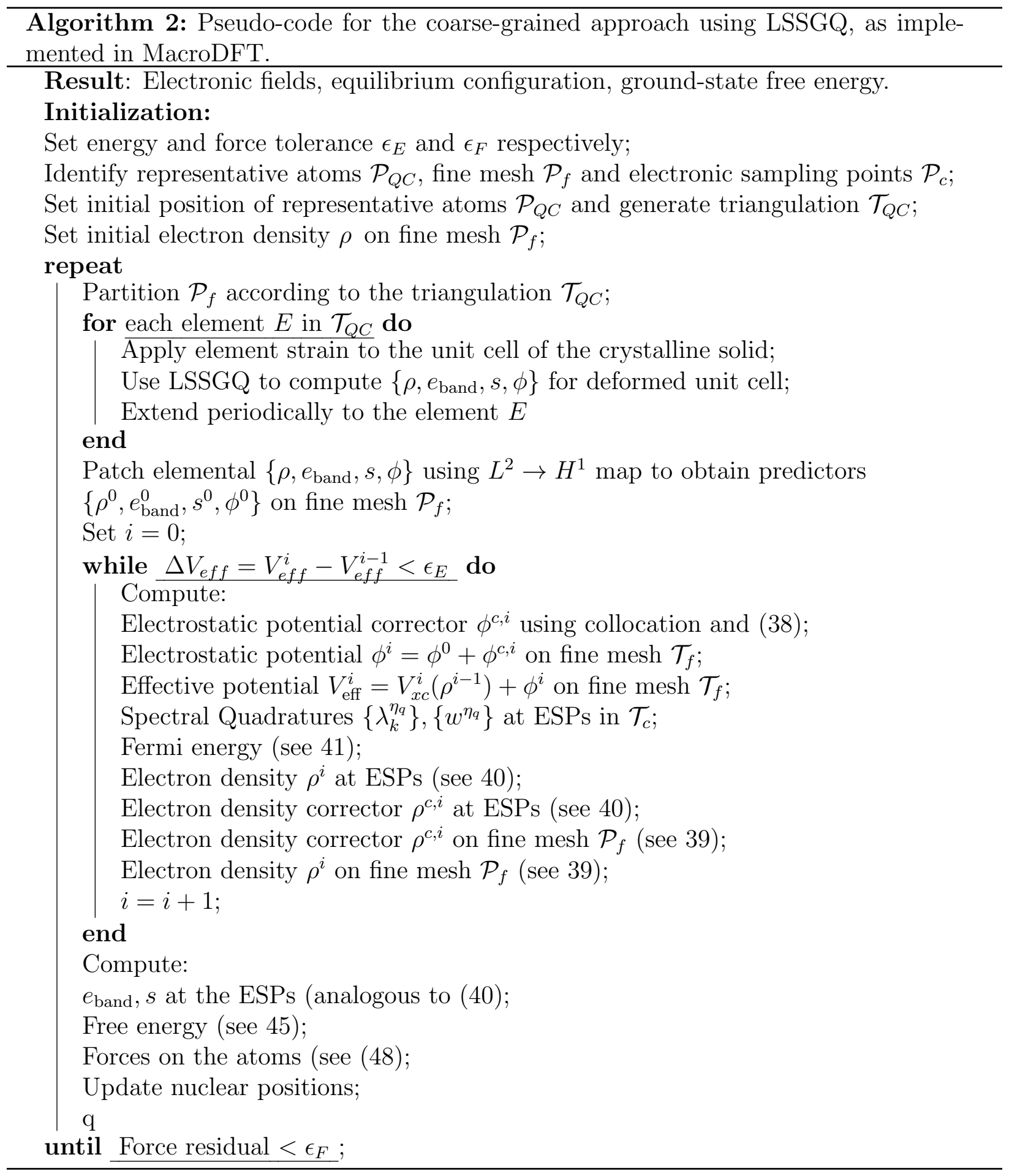




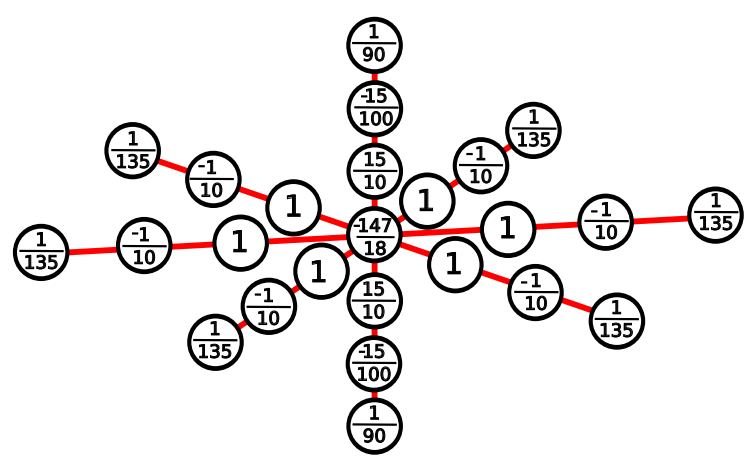

Figure 3: Sixth-order finite-difference stencil adapted to the HCP structure of Mg. The number inside the spheres indicates the finite-difference coefficient for computing the Laplace operator.

\section{MacroDFT: Implementation at Full Resolution}

\subsection{Implementation}

We have implemented and studied the LSSGQ method with Hexagonal-Closed-Packed Magnesium as a test case. We use a real space Bulk-derived Local Pseudopotential (BLPS) [36]. The Laplace operator is evaluated using a $6^{\text {th }}$ degree finite-difference scheme that combines a triangular stencil on constant- $z$ planes and a conventional stencil in the $z$-direction [41]. Figure 3 shows the resulting stencil. In order to streamline the calculations, neighbor-lists are maintained and the shape functions associated to the neighbor list are stored in a global hash table. The Poisson equation is solved using the Generalized Minimal Residual method (GMRES) [42]. The Fermi energy is obtained through a combination of a bisection method and a Newton-Raphson iteration with cubic line search using the PETSc library [43]. The SCF iteration is accelerated using the Broyden multisecant method combined with mixing, as proposed by Fang and Saad [44]. The energy-convergence tolerance is set to $10^{-5} \mathrm{eV}(0.01$ $\mathrm{meV})$. The equilibrium configurations of the nuclei are obtained by energy-minimization using a non-linear conjugate gradient [45] based on the force evaluation described in the Appendix. The force-convergence tolerance is set to $10^{-3} \mathrm{eV} / \AA$ per atom. These values are consistent with those used in other finite-difference methods [3] and plane wave methods. Finally, we choose $\sigma=0.8 \mathrm{eV}$ which corresponds to a temperature of $T=10,000 \mathrm{~K}$ unless mentioned otherwise and extrapolate to find the ground-state energy at absolute zero using 20. We have taken this value to be consistent with other studies of metals (for example, [46, 47] take $\sigma=1.0 \mathrm{eV}$ ). The implementation is parallelized using a domain decomposition technique and the Message Passing Interface (MPI) library in combination with threads using OpenMP. 


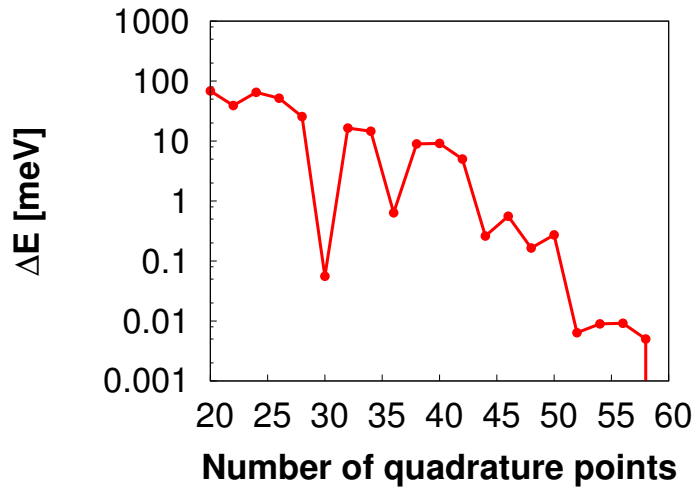

(a)

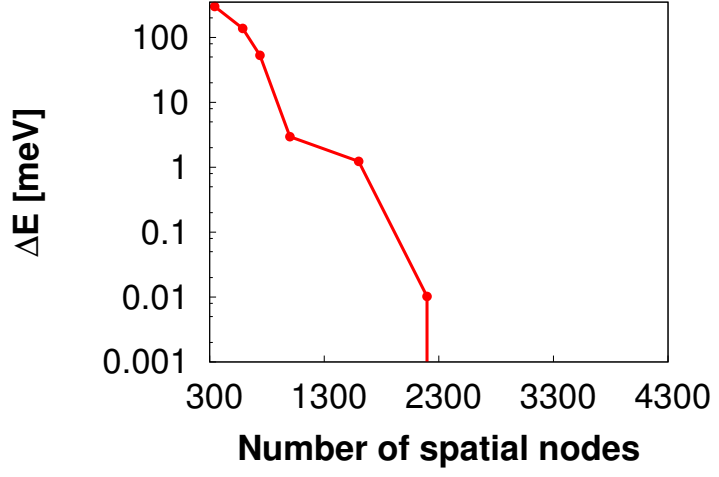

(b)

Figure 4: Convergence of Mg cohesive energy per atom with (a) spectral and (b) spatial resolution. $\Delta E$ reference to the difference between the energy computed with the current parameter and that computed with the largest parameter in the plot.

\subsection{Convergence and verification}

\subsubsection{Bulk properties}

We begin by assessing the convergence characteristics of LSSGQ with respect to spectral quadrature and spatial resolution. Figures $4 \mathrm{a}$ and $4 \mathrm{~b}$ specifically show the convergence of the cohesive energy per atom of $\mathrm{Mg}$ for calculations performed using periodic boundary conditions on the unit cell containing two atoms. The calculations do indeed exhibit convergence with respect to the number of quadrature points and the spatial resolution. Figure 4 a shows the error in cohesive energy computed with various quadrature points compared with the result with 60 quadrature points (These calculations are performed for 2200 spatial nodes). Similarly, Figure $4 \mathrm{~b}$ shows the error in cohesive energy computed with various spatial resolution compared with the result with 4300 nodes (These calculations are performed for 40 quadrature points). We obtain accuracies of $0.01 \mathrm{meV}$ or better. We then compare the results obtained with the LSSGQ method and other DFT simulation using the same pseudopotential [36] in Table 1 to find good agreement. The bulk modulus was calculated by fitting the energy versus volume curve to the Murnaghan equation of state [48]. Note that we have to account for the anisotropy of the material optimizing $c / a$ for each volume. Further, we find $a=3.109 \AA$ and $c / a=1.626$, and these are consistent with values previously obtained with other ab-initio calculations with other pseudopotentials [49, 36, 50] (e. g. $a=3.11 \AA$ and $c / a=1.624)$.

\subsubsection{The role of temperature}

We introduced temperature to obtain a smooth occupancy function (24) that can be approximated using polynomials. The choice of this temperature is delicate: it has to be small enough to be physically meaningful but large enough to enable accurate approximation. We study this using the cohesive energy of $\mathrm{Mg}$.

\footnotetext{
${ }^{1}$ The treatment is readily modified for a more general situation
} 
Table 1: Comparison between the bulk modulus $\left(B_{0}\right)$, minimum energy in the periodic unit cell $\left(E_{\text {min }}\right)$ and the equilibrium volume $\left(V_{0}\right)$ for Mg obtained with LSSGQ method and traditional DFT using the same pseudopotential using ABINIT [36].

\begin{tabular}{ccc}
\hline \hline & LSSGQ & DFT ( ABINIT, [36] ) \\
\hline$B_{0}$ & $38.75 \mathrm{GPa}$ & $38.4 \mathrm{GPa}$ \\
$E_{\min }$ & $-24.671 \mathrm{eV}$ & $-24.678 \mathrm{eV}$ \\
$V_{0}$ & $42.317 \AA^{3}$ & $42.351 \AA^{3}$ \\
\hline \hline
\end{tabular}

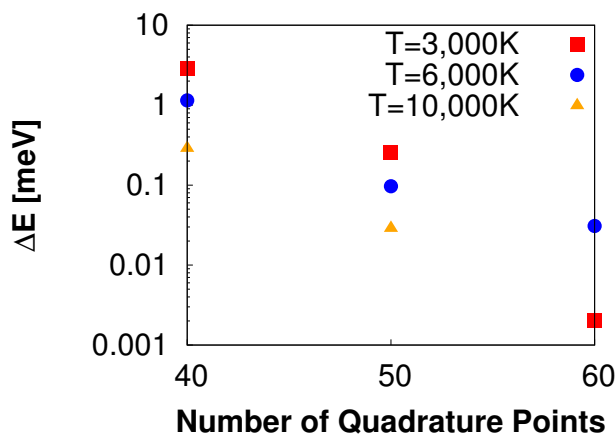

(a)

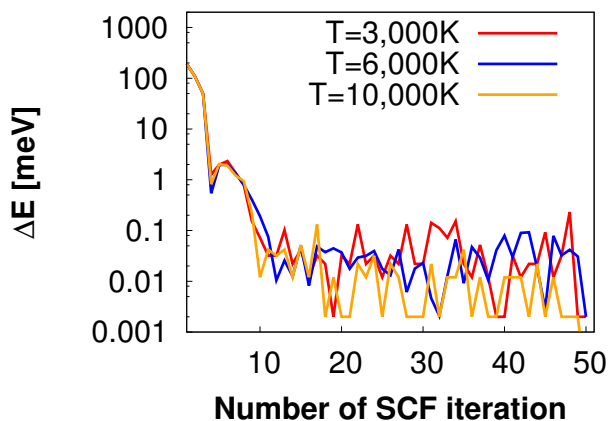

(b)

Figure 5: Interplay of temperature, number of quadrature points and self-consistent iteration. (a) The difference in cohesive energy per atom with the number of quadrature points for various temperatures. (b) The difference in cohesive energy with self-consistent iteration for various temperatures with $\mathrm{K}=60$. We take the calculation at $T=10,000 K$ with $K=60$ as reference.

We compute the cohesive energy for $\mathrm{Mg}$ using the previous set up for a different three different temperatures $-T=3,000 \mathrm{~K}, T=6,000 \mathrm{~K}$ and $T=10,000 \mathrm{~K}(\sigma=0.26 \mathrm{eV}, \sigma=0.52$ $\mathrm{eV}$ and $\sigma=0.86 \mathrm{eV}$ respectively), with each case computed with three different number of quadrature points $K=40, K=50$ and $K_{3}=60$. The results are shown in Figure 5 a as the difference between the cohesive energy per atom computed a given temperature and given number of quadrature points from that at computed using $T=10,000$ and $K=60$. We first observe that the values computed at $T=3,000 \mathrm{~K}, T=6,000 \mathrm{~K}$ and $T=10,000 \mathrm{~K}$ agree (up to order $0.01 \mathrm{meV}$ ) when we use $K=60$ quadrature points. Thus, for this example, the three temperatures give the same result when resolved sufficiently. Second, we observe that at $T=10,000 K$, the computed value changes very little for $K=40$, $K=50$ and $K=60$. In contrast, at $T=3,000$, we see large differences at $K=40$ and need $K=60$ to obtain reasonable values. Since the occupancy function is sharper at lower temperatures, one needs a large number of quadrature points to accurately compute it. In contrast, a higher temperature enables computation with fewer quadrature points. We also see in Figure $5 \mathrm{~b}$ that the cohesive energy per atom oscillates more at lower temperatures with the self-consistent iterations.

We conclude that $T=10,000 \mathrm{~K}(\sigma=0.86 \mathrm{eV})$ is adequately low for a physically meaningful result and enables stable computation at reasonable resolution $K=40$ for this example. 


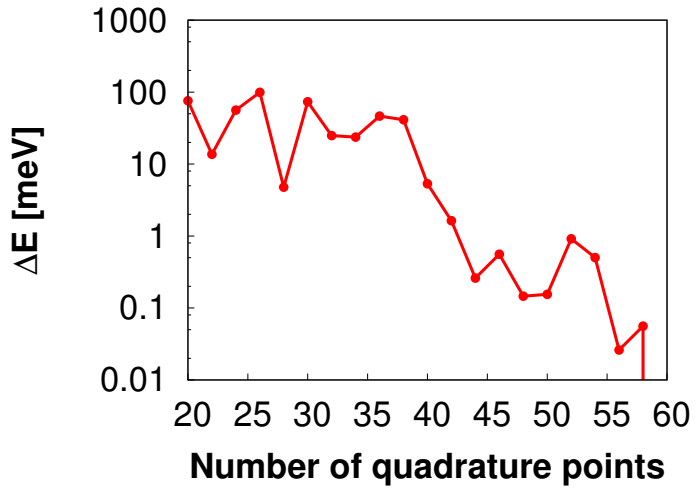

(a)

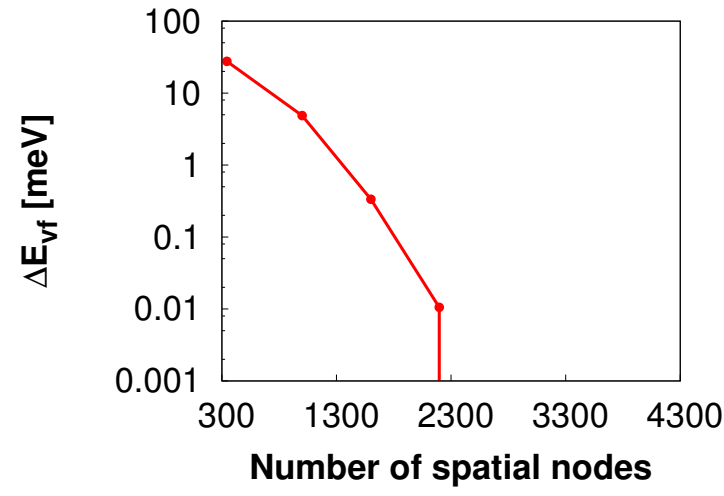

(b)

Figure 6: Convergence of vacancy formation energy with (a) spectral and (b) spatial resolution. $\Delta E$ reference to the difference between the energy computed with the current parameter and that computed with the largest parameter in the plot.

\subsubsection{Vacancy formation energy}

As an additional test, we calculate the vacancy formation energy for a $\mathrm{Mg}$ single crystal with fields attaining bulk values at the boundary. The representative volume is a cuboid of dimensions $6 a_{0} \times 6 a_{0} \times 6 c_{0}$. The calculations are carried out using a fine discretization of the unit cell using about 2200 nodal points. The value of the vacancy formation energy can be computed as 29]

$$
E_{v f}(M)=M E_{\min }-\frac{M}{M-1} E(M-1)
$$

where $M$ is the number of atoms in the perfect crystal and $E(M-1)$ is the energy stored in the crystal with the vacancy. The energy of the prefect crystal is approximated by $E(M)=$ $M E_{\text {min }}$ where $E_{\text {min }}$ is the energy per atom obtained in the periodic unit cell calculation.

Figure 6a and Figure 6b show the convergence of the vacancy formation energy with spectral resolution and the convergence with respect to spatial discretization. For more than 40 quadrature points and 2200 spatial nodes per unit cell, the change in the energy is below than $5 \mathrm{meV}$. The computed value of the vacancy formation energy, with a computational cell of 432 atoms, is $E_{f}(M)=0.79 \mathrm{eV}$, which is in good agreement with previously computed values, $E_{f}(M)=0.74-0.83 \mathrm{eV}$ [36, 51, 49], and with experimental values, $E_{f}(M)=0.58-$ $0.9 \mathrm{eV}$, cf. [52, 53, 54, 55].

Additionally, we compare the forces on atoms using the expression in the Appendix and the values obtained by numerical differentiation of the ground state energy. For the latter, the atoms are perturbed from their position by $\Delta x_{i}=0.0005 \AA$ in each direction $i=1,2,3$, and the forces are obtained numerically using a 2-point finite-difference formula [41. We find that the forces computed using the two methods have identical direction (up to numerical resolution) and magnitude that differs by less that $1.25 \%$.

Finally, we find that the atomic displacement. The maximum value is encountered for the atom nearest to the vacancy with a magnitude of the displacement about $1 \% a_{0}$. This is in good agreement with the displacement field reported by Chetty et al. [49] who found 


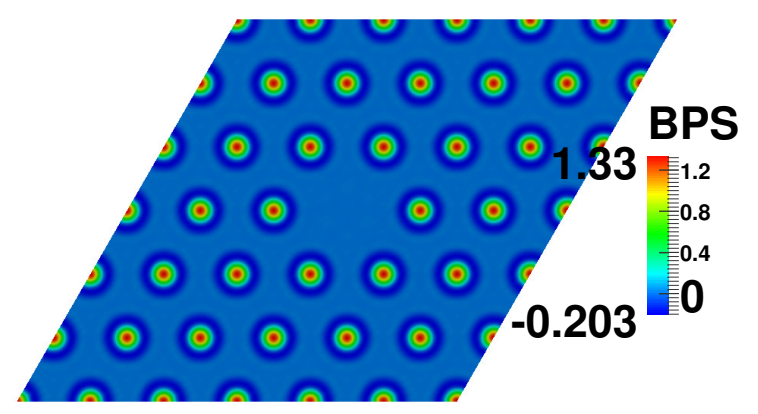

(a)

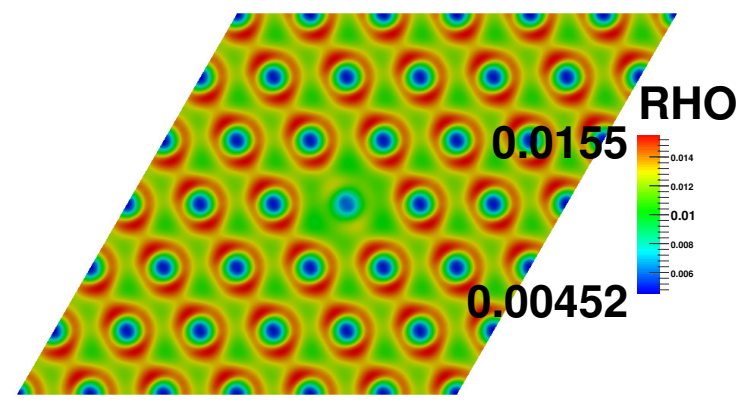

(c)

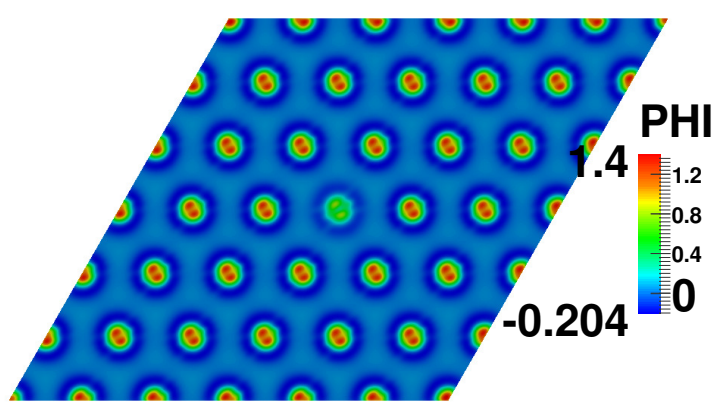

(b)

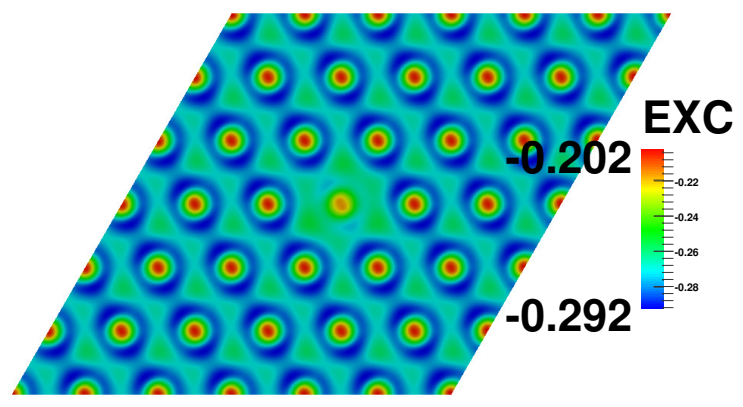

(d)

Figure 7: Vacancy in HCP Mg. Various fields through the plane $z=0$ (BPS: Nuclear and local psudopotential charge density in Bohr ${ }^{-3}$, PHI: Electrostatic potential in Hartree/electronic charge, RHO: Electron density in Bohr ${ }^{-3}$, EXC: derivative of the exchange-correlation in Hatree/electronic charge). Computational cell containing 432 atoms $\left(6 a_{0} \times 6 a_{0} \times 6 c_{0}\right)$, with bulk values enforced at the boundary for all fields. 


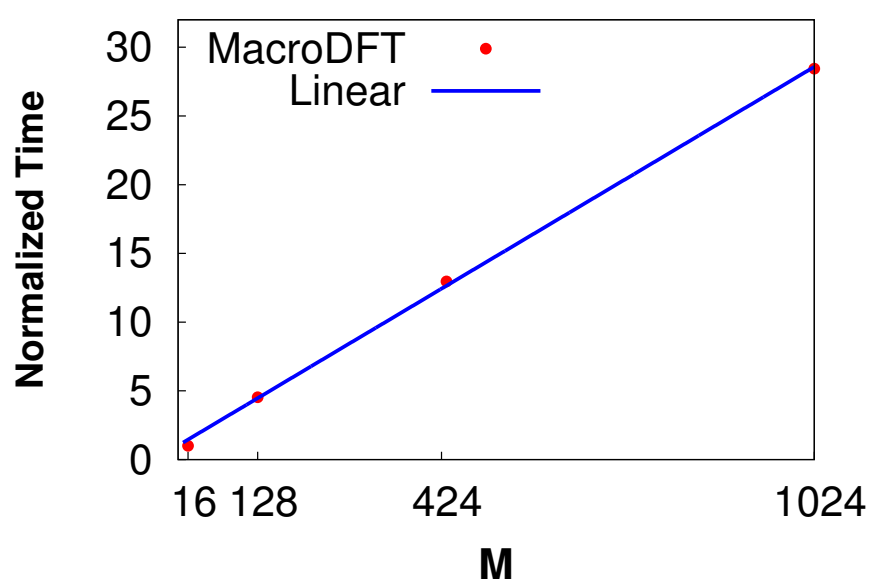

Figure 8: Linear scaling with number of atoms for simulation of vacancy in HCP-Mg in specimens up to 1024 atoms. Normalized time is the ratio of computational time for a cell with $M$ atoms to that of a computational cell with 16 atoms.

$1.1 \% a_{0}$ using the Troullier-Martins non-local pseudo-potential [37].

Figure 7 shows the total charge density, total electrostatic potential, electron density and the first variation of the exchange-correlation energy on a section of the crystal through the plane $z=0$ and for the relaxed solution.

\subsection{Linear scaling and parallel performance}

We demonstrate the linear scaling property of LSSGQ by studying the HCP-Mg vacancy just described over a range of cell sizes, with numbers of atoms ranging from 16 to 1,024 and numbers of nodes ranging from 15,625 to 912,673. All tests were carried out on MIRA IBM BG/Q 1.6 GHz PowerPC A2 supercomputer property of Argonne National Laboratory. Figure 8 shows the normalized time for force evaluation as a function of problem size. As may be seen from the figure, almost perfect linear scaling is achieved over the range of problem sizes considered.

Next, we assess the parallel performance for different number of processors. In our hybrid implementation, we first divide tasks between the different MPI nodes available and subsequently start a set of threads using OpenMP. MPI communication occurs only when the thread tasks are completed. This approach increases communication between processors in the same node and decreases the communication between nodes, thus avoiding MPI barriers or bottlenecks. The number of threads started can be changed in order to obtain an optimal combination between MPI tasks and threads.

To obtain the optimal combination, we have run the same problem, a cell with 1,024 atoms and 912,673 ESPs, using different numbers of threads per node, ranging from 1 thread (pure MPI) to 64 threads per node. As may be seen in Figure 9a, neither pure MPI (black bars labeled as MPI-only) nor pure threading provide good performance. We find good performance for the combination of 8 MPI tasks and 8 threads. We also find good parallel performance (approximately $93 \%$ up to 1,024 processors in 64 nodes) for this combination. 


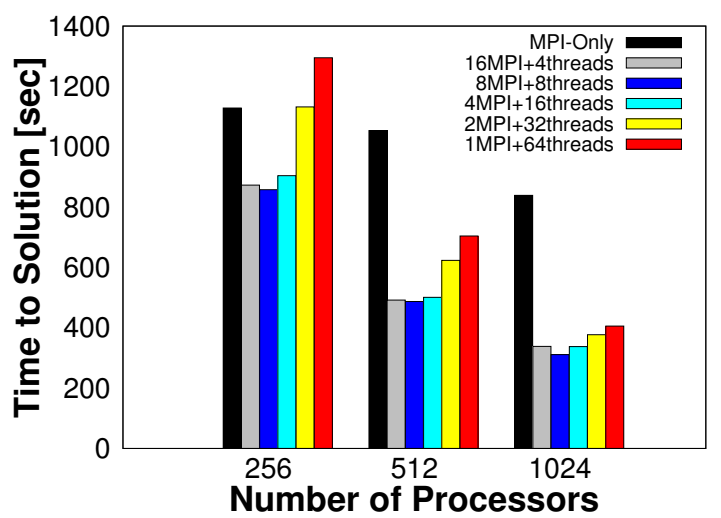

(a)

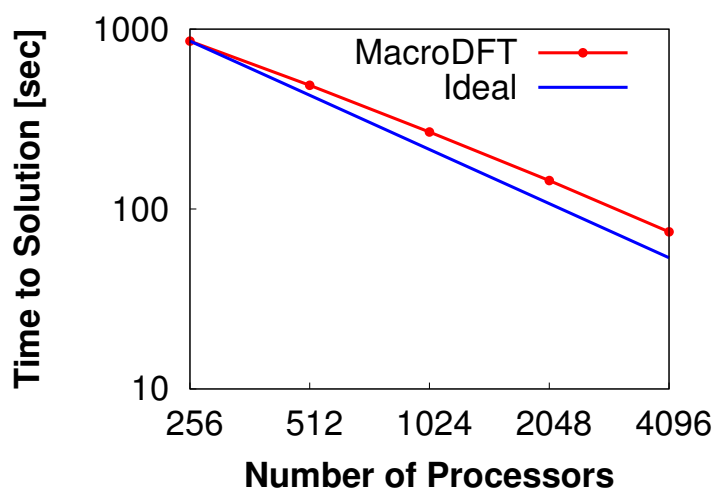

(b)

Figure 9: Parallel performance evaluated on vacancy in HCP-Mg with a cell containing 1024 atoms and 912,673 ESPs. (a) Performance for different ratios of MPI tasks to threading. The combination of 8 MPI tasks and 8 threads per node gives the optimal performance and scaling up to 64 nodes with 1024 cores. (b) Parallel scaling with 8 MPI tasks and 8 threads per node.

Interestingly, as the number of processors increases, the gap between the different number of threads is reduced. Finally, Figure 9b shows the parallel performance for different number of processors for the previous simulation cell containing 1,024 atoms. For these tests, we have used the optimal combination of 8 MPI tasks and 8 threads per node. The speed-up and the time to solution show a linear trend and the relative speed-up for 4,096 processors is approximately $75 \%$.

\section{MacroDFT: Implementation with coarse-graining}

We now demonstrate, validate and assess the implementation of the coarse-graining as described in Section 4 in MacroDFT. In this section, we consider a single vacancy located at the center of a computational cell of variable size. The computational cell is terminated by enforcing bulk conditions on all fields at the boundary of the cell. Given the simplicity of the example, the predictor field is taken to be the electronic fields of the bulk materials. Based on a standard error analysis for the Laplace equation, the element-size distribution scales as $\mathcal{O}\left(r^{6 / 5}\right)$ with distance $r$ to the vacancy. We construct all triangulations by Delaunay triangulation using the library Tetgen [56]. For all simulations, we set up the number of quadrature points to $40, \sigma=0.8 \mathrm{eV}$, the energy-convergence tolerance is set to $10^{-5} \mathrm{eV}$ and the force-convergence tolerance is set to $10^{-3} \mathrm{eV} / \AA$.

\subsection{Convergence with respect to number of ESPs}

Figure 10 shows the formation energy as a function of the number of ESPs for a crystal of 93,312 atoms and $81 \times 10^{6}$ finite-difference nodes. Recall that ESPs or electronic sampling points $\mathcal{P}_{c}$ are a subset of the finite-difference nodes $\mathcal{P}_{f}$ on which the electronic fields are sampled in the coarse-grained approach. We observe from the figure that the energy con- 


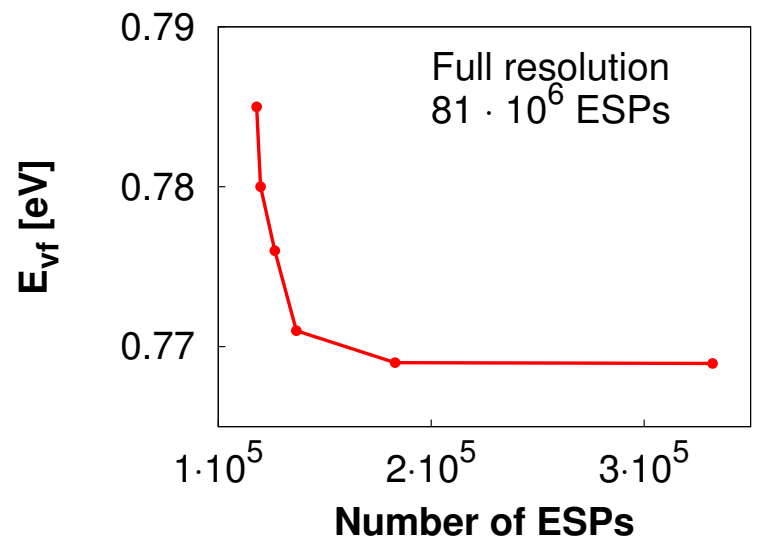

Figure 10: The efficacy of coarse-graining. Convergence of the formation energy with the number of electronic sampling points (ESPs) for a computational cell containing 93,312. Note that the energy converges at around $1.8 \times 10^{5}$ ESPs giving a speed up of 443 in this example.

Table 2: The efficacy of coarse-graining and the convergence with respect to cell size. List and details of cases considered. The cell size is $n \times n \times n$ unit cells.

\begin{tabular}{ccccccc}
\hline \hline Case & $n$ & Atoms & RepAtoms & ESPs & Speed-up & $E_{v f}$ \\
\hline 1 & 12 & 3,456 & 1109 & 150,353 & 20 & 0.773 \\
2 & 36 & 93,312 & 3156 & 183,057 & 443 & 0.769 \\
3 & 108 & $2.5 \times 10^{6}$ & 9275 & 215,761 & 10,112 & 0.768 \\
4 & 972 & $1.8 \times 10^{9}$ & 118428 & 562,338 & $2,822,648$ & 0.768 \\
\hline \hline
\end{tabular}




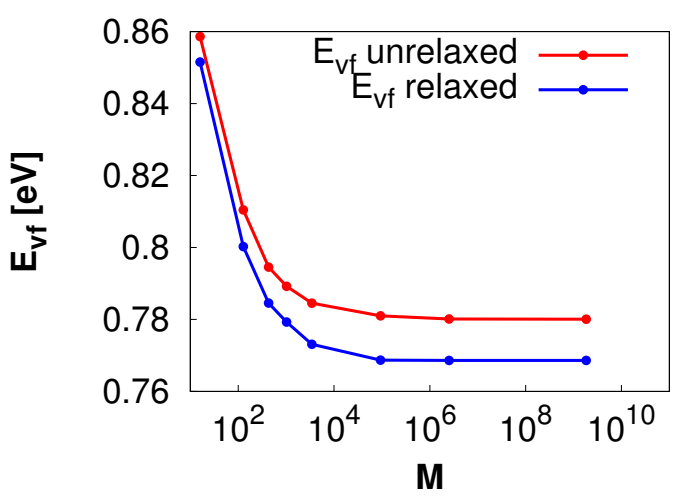

(a)

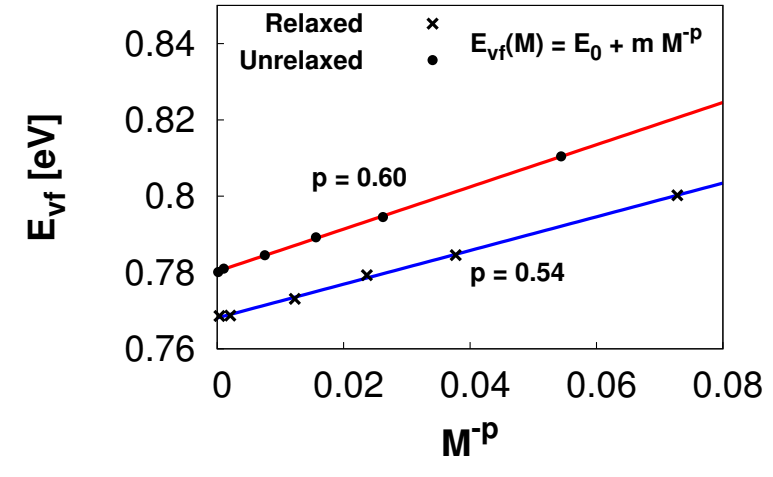

(b)

Figure 11: Convergence of the vacancy formation energy vs. computational cell-size shown through (a) number of atoms and (b) inverse power of the number of atoms. The coefficients of the power law $E_{v f}=E_{0}+m M^{-p}$ that best fit the data are: $E_{0}=0.780, m=0.55, p=0.60$ for the unrelaxed case and $E_{0}=0.768, m=0.4417, p=0.54$ for the relaxed case.

verges at $\sim 1.8 \times 10^{5}$ ESPs. The speed-up achieved by the CG approach, defined as the ratio of the size of the finite-difference grid (fully-resolved LSSGQ) to the number of ESPs, is approximately 440. This ratio becomes larger with larger systems as shown in Table 2 .

\subsection{Convergence with respect to cell size}

We proceed to study the convergence of the vacancy formation energy with respect to the cell size. To this end, we carry out simulations involving different number of atoms and the details are listed in Table 2. For all simulations, the number of ESPs is selected so that the vacancy formation energy is numerically converged. As stated earlier, the speedup increases with cell size. In particular, the speed-up factor of over a million that is obtained for the largest cell of a billion atoms is remarkable.

Figure 11a shows the dependence of the vacancy formation energy on cell size of $M$ atoms, both for simulations where the atomic positions are held fixed at the initial guess (unrelaxed) and for simulations where the atomic positions are relaxed. We see that the vacancy formation energy converges slowly with the number of atoms, for specimens where the position of the atoms are held fixed (at positions corresponding to a perfect crystal) as well as for specimens where the position of the atoms is relaxed. We see from Figure $11 \mathrm{~b}$ that the formation energy scales as a power of the number of atoms. The coefficients of the power law $E_{v f}=E_{0}+m M^{p}$ that best fit the data are: $E_{0}=0.780, m=0.55, p=0.60$ for the unrelaxed case and $E_{0}=0.768, m=0.4417, p=0.54$ for the relaxed case.

Figure 12 shows the corrector electrostatic field (i. e., the difference between the actual electrostatic field and that in a perfect crystal) on the basal and prismatic planes near the vacancy for the $2.5 \times 10^{6}$-atom crystal with the atomic positions fixed (a,c) and relaxed (b,d). Note that even in the unrelaxed specimen (Figure 12 a,c), one sees Friedel-type oscillations that extend over a number of atomic spacings. This is the reason one needs a specimen of 


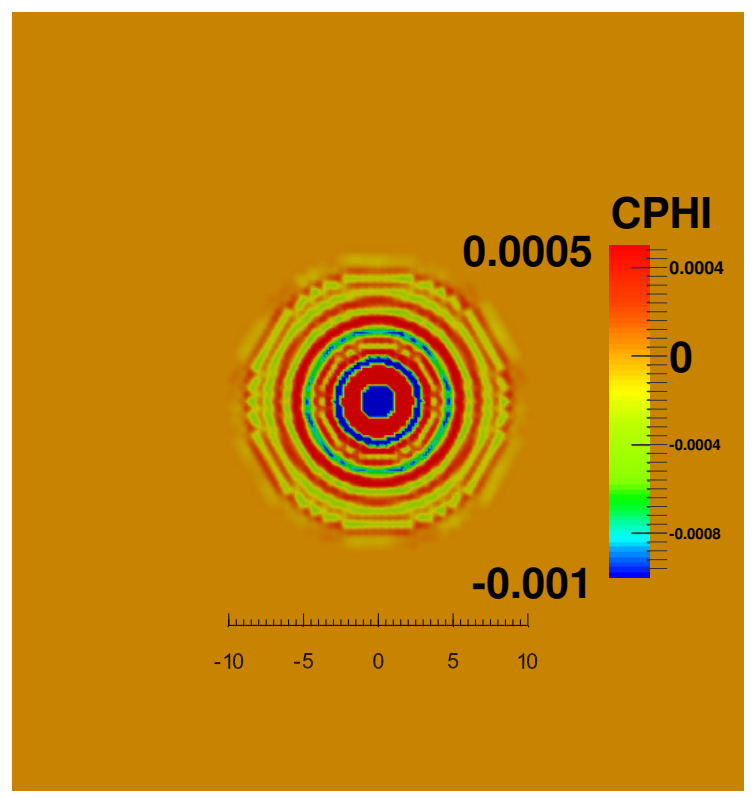

(a)

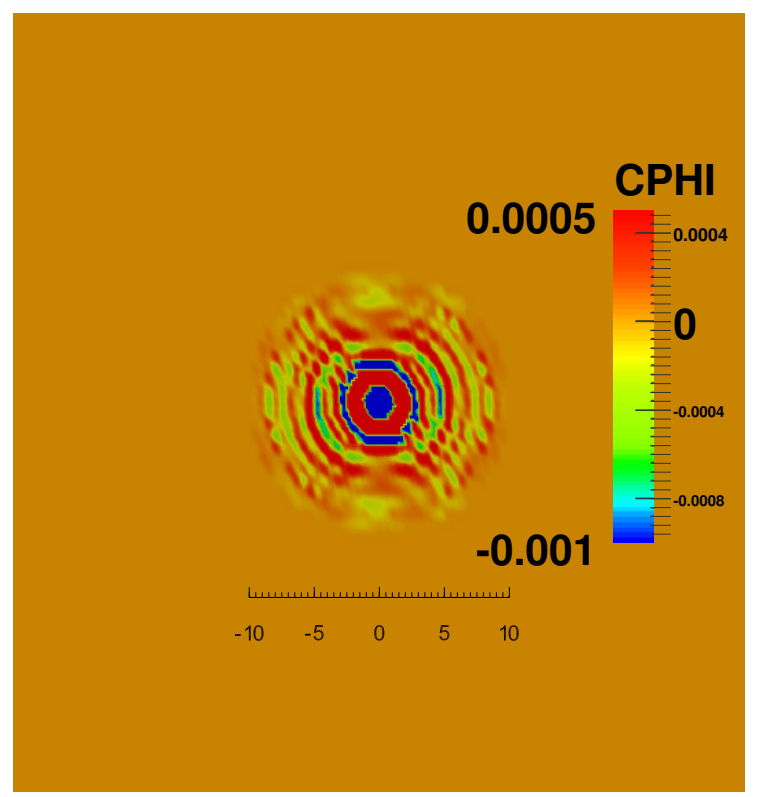

(c)

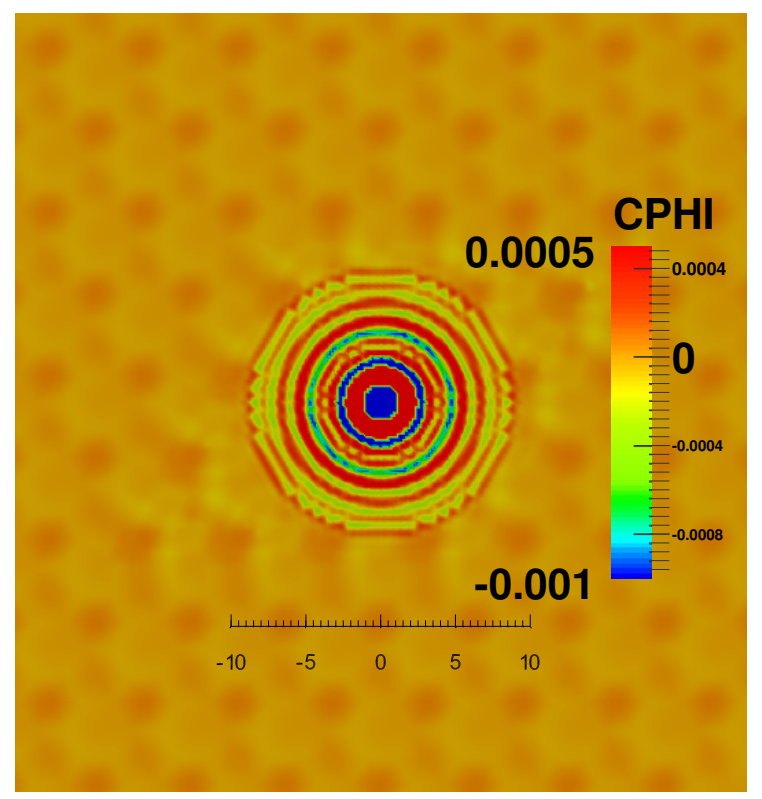

(b)

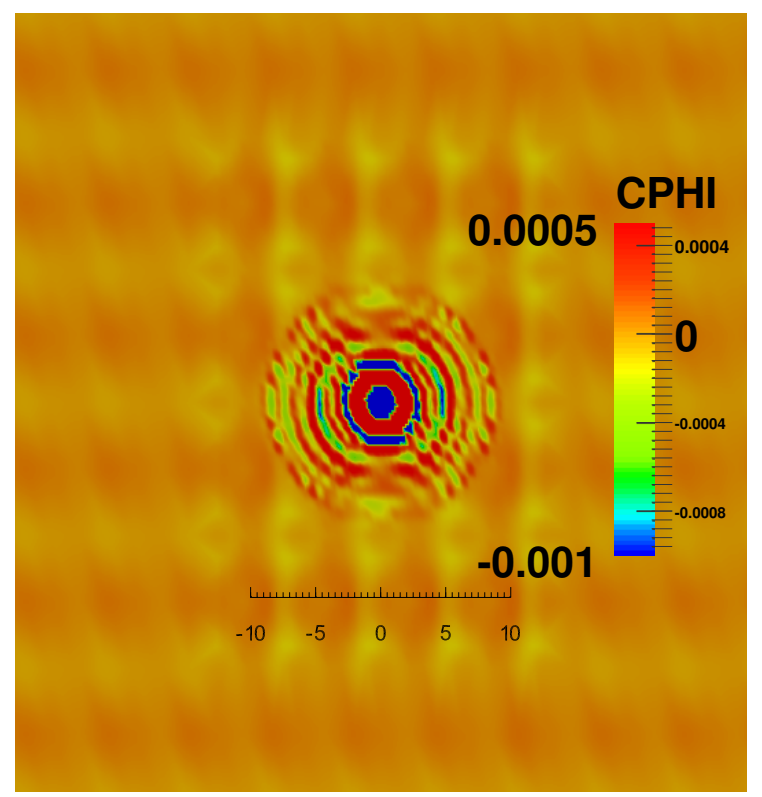

(d)

Figure 12: The corrector electrostatic potential (deviation from that of a perfect crystal) in a $\mathrm{Mg} 2.5 \times 10^{6}$-atom specimen with a single vacancy in the vicinity of the vacancy. (a,c) Basal and prismatic planes with positions of the atoms fixed at those of a perfect crystal. (b,d) Basal and prismatic planes with positions of the atoms relaxed. The inset length-scale bar is in Bohr, and the potential in Hartree/electronic charge. 
Table 3: Comparison of the vacancy formation energy in eV computed with MacroDFT with experiment and prior DFT calculations. The cell size in terms of number of atoms used for each calculation is indicated in parenthesis.

\begin{tabular}{|c|c|c|c|c|c|}
\hline Expt & MacroDFT $^{\mathrm{a}}$ & DFT(CASTEP) ${ }^{a}$ & $\operatorname{DFT}(\mathrm{ABINIT})^{\mathrm{b}}$ & $\mathrm{DFT}^{\mathrm{b}}$ & $\operatorname{DFT}(\mathrm{PWP})^{\mathrm{b}}$ \\
\hline $0.81^{52]}$ & $0.851(16)$ & $0.822(36)^{[36}$ & $0.802(36)^{36}$ & $0.91(36)^{49}$ & $0.80(36) \sqrt{51}$ \\
\hline $0.58^{[53]}$ & $0.800(128)$ & & & $0.83(96)^{[49]}$ & $0.74(96)$ [51] \\
\hline $0.79^{[\underline{54}]}$ & $0.785(432)$ & & & & \\
\hline $0.90^{[55]}$ & $0.779(1,024)$ & & & & \\
\hline & $0.773(3,056)$ & & & & \\
\hline & $0.769(93,312)$ & & & & \\
\hline & $0.768(2,519,424)$ & & & & \\
\hline & $0.768\left(1.8 \times 10^{9}\right)$ & & & & \\
\hline
\end{tabular}

a All calculations were performed with BPLS pseudo-potential [36].

b All calculations were performed with Troullier-Martins non-local pseudo-potential [37].

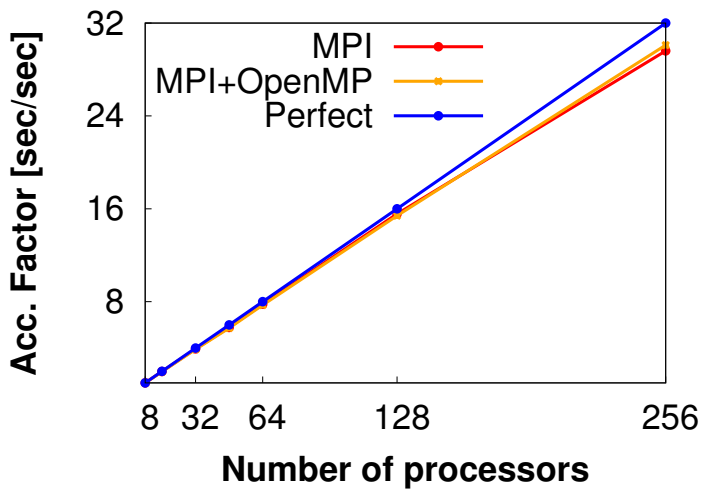

(a)

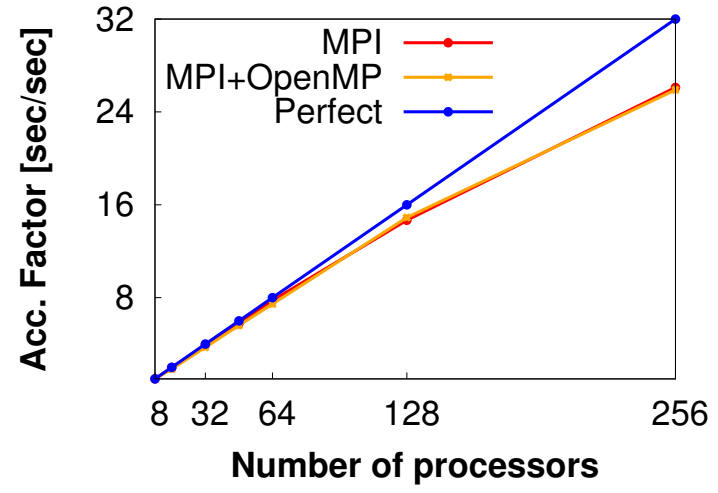

(b)

Figure 13: Parallel performance of MacroDFT on an Intel Xeon ${ }^{\circledR}$ E5345-2.33GHz Quad-Core cluster: a) Dynamic load balance. b) Spatial domain decomposition.

about 1000 atoms (a simulation cell of about $10 a_{0} \times 10 a_{0} \times 10 c_{0}$ ) for convergence even with the atomic positions held fixed. Once the atomic positions are relaxed, we see that the region with the Friedel type oscillations grow and there are small perturbations that extend even further since atomic displacements decay slowly. Importantly, atomistic displacements defeat the short-sightedness of electronic matter [57]. Therefore, we see even slower convergence of the vacancy formation energy with respect to the cell size.

Finally, we compare our results with experiments and previous DFT calculations in Table 3. The experimental values have a wide spread. The MacroDFT calculations show a systematic decrease of the formation energy with cell size. The values we obtain with smaller cells are consistent those obtained by other DFT studies. 


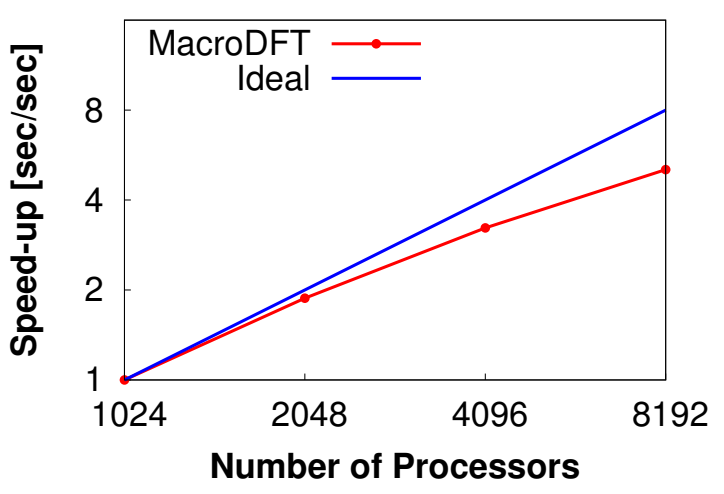

(a)

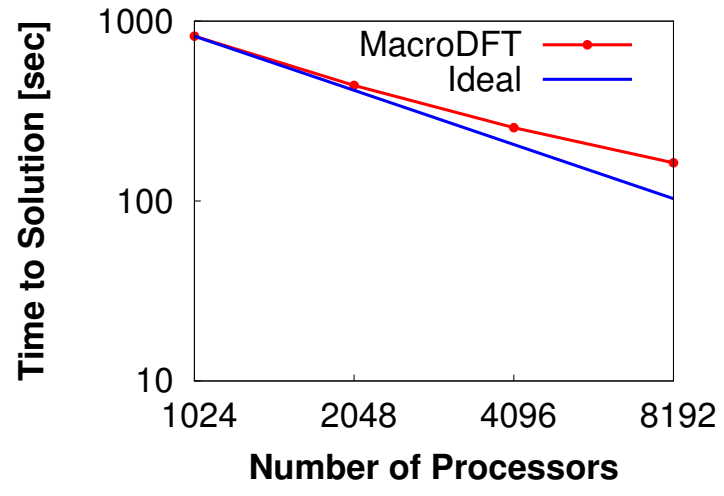

(b)

Figure 14: Parallel performance of MacroDFT for a large scale simulation on MIRA IBM BG/Q 1.6 GHz PowerPC A2: a) Speed-up. b) Time to solution.

\subsection{Parallel performance}

We now assess the parallel performance and scaling of MacroDFT with coarse graining using the case of $2.5 \times 10^{6}$ atoms (case 3 of Table 2 ) on two different High Performance Computing (HPC) platforms.

We first consider a modest Intel Xeon ${ }^{\circledR}$ E5345-2.33GHz Quad-Core with 256 processors. We follow two different parallelism paradigms, spatial domain decomposition (SDD) and dynamic load balancing (DLB) where one thread is used to distribute the work between the available nodes. Figure 13 shows that DLB achieves better performance for both MPI and hybrid programming. The slow-down of SDD with increasing number of processors may be traced to imbalances in the number of operations required by ESPs in the fullyresolved and coarse-grained regions. DLB overcomes this since each node can consider ESPs which may belong either to the fully-resolved or the coarse-grained regions. Further, we observe that for 128 processors and beyond, the hybrid approach achieves equal or better performance than MPI due to the reduction of inter-node communications, which are slower than memory access. Although the differential is modest at 256 processors (from 29.6 to 30.1 ), it is expected to be grow for larger and increasing numbers of processors.

Next, we consider the large-scale MIRA IBM BG/Q 1.6 GHz PowerPC A2. Figure 14 shows the relative speed-up using the simulation with 1024 processors as reference and time to the solution for a number of processors ranging from 1,024 to 8,192 using the DLB algorithm. For these tests, we have used the optimal combination of 8 MPI tasks and 8 threads per node. The performance achieved by MacroDFT for coarse-graining problems is notable, reaching about $75 \%$ of efficiency for 8,192 processors.

\subsection{Sublinear scaling}

Figure 15 shows the sublinear scaling of clock-time as a function of crystal size. The scaling is well-represented by a power-law relation of the type $t=b M^{A}$ with power $A=0.05$ and pre-factor $b=5.06$. This extremely sublinear performance is a result of the simplicity of the 


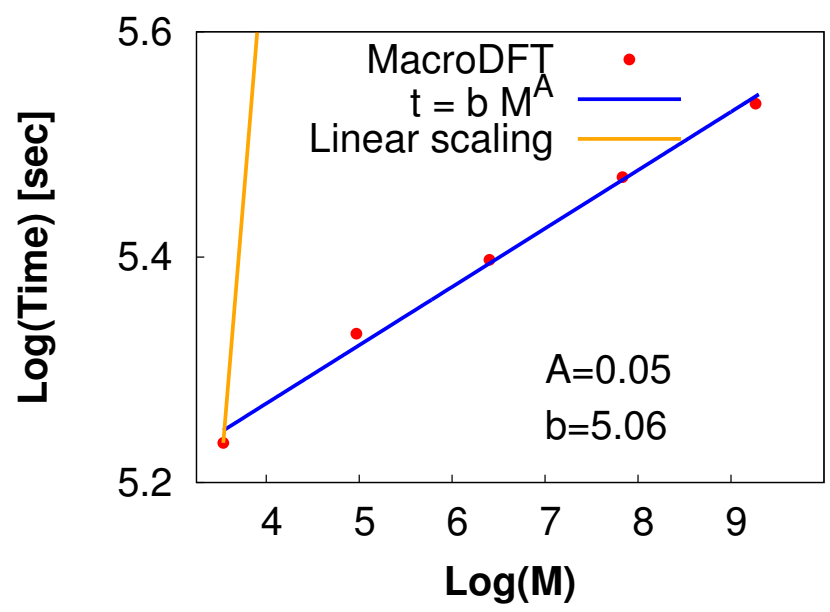

Figure 15: Vacancy in Mg. $2.5 \times 10^{6}$ crystal and coarse-grained model consisting of $\sim 5 \times 10^{3}$ representative atoms and $\sim 2.16 \times 10^{4}$ ESPs. Clock-time scaling with crystal size measured in terms of number of atoms.

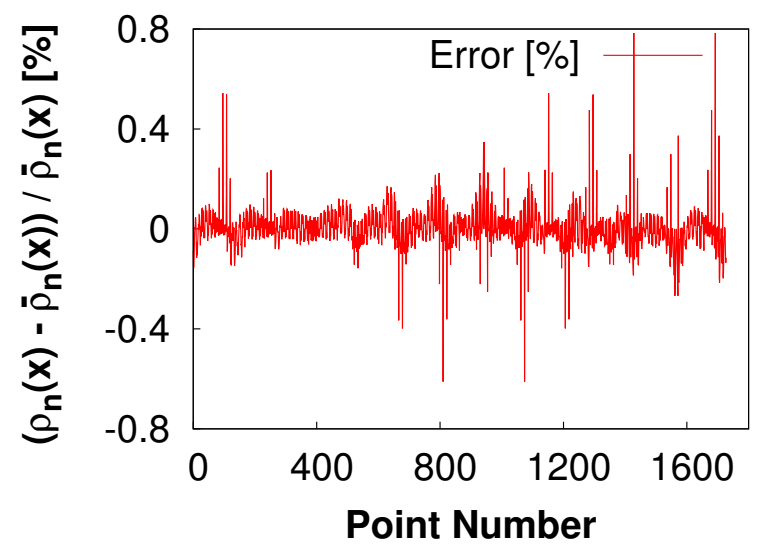

Figure 16: Accuracy of the coarse-graining approximation. Percentage error between the exact electron density $\bar{\rho}$ and the coarse-grained approximation $\rho$ at 1800 points in the fine grid.

examples considered here. For more complex examples with slower decay of elastic fields, we expect the gains to be more modest, but still significantly better than linear.

\subsection{Validation of the Coarse-graining approach}

We now endeavor to validate the coarse-graining approach used in this work. We consider the problem of a HCP-Mg vacancy with $2.5 \times 10^{6}$ atoms (case 3 of Table 2). We then consider a domain of size $a_{0} \times a_{0} \times c_{0}$ centered at $\left[9 a_{0}, 0,0\right]$ in the coarse-grained region. This region has about 1800 points on the fine grid that are not ESPs. We compute the 'exact' electronic fields at these fine grid points using LSSGQ and compare it with the those obtained from our 


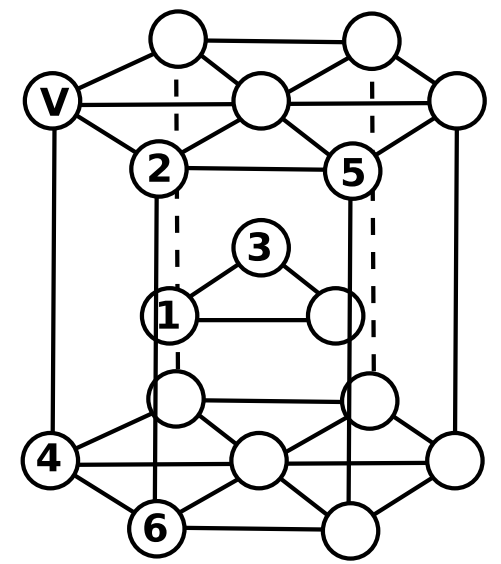

(a)

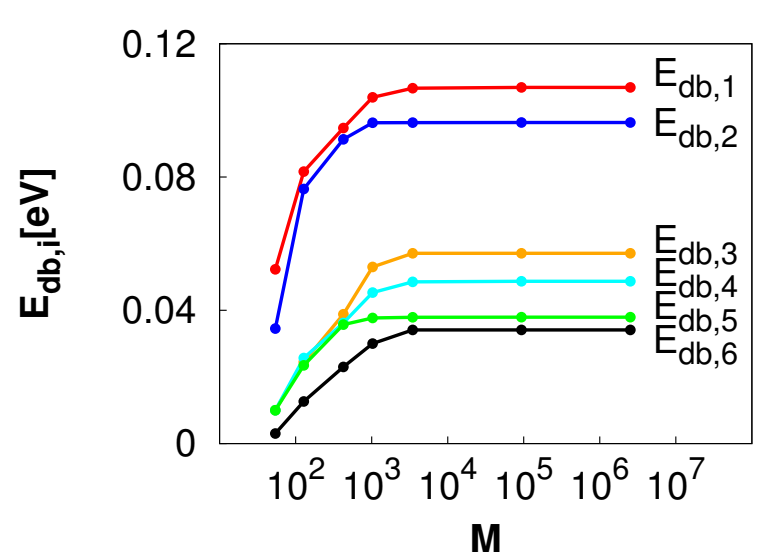

(b)

Figure 17: Divacancy formation energy. (a) Various divacancy complexes studied. The missing atoms are at $\mathrm{V}$ and position labeled $i=1, \ldots 6$ for first to sixth nearest neighbor. (b) Divacancy binding energy vs. cell size for the six configurations. Note that the binding energy orders with neighbor distance.

coarse-grained representation 2 , Figure 16 shows the error between the exact charge density $\bar{\rho}$ and that obtained using our coarse-graining scheme $\rho$ at all $\sim 1800$ points. The point wise error is $0.8 \%$ or below. We also find that the average error $\left(1 / N \sum_{n=1}^{N}\left(\rho_{n}(\mathbf{x})-\overline{\rho_{n}}(\mathbf{x})\right)\right)$ is $5.0 \times 10^{-7}$ and the root mean square error $\left(\sqrt{1 / N \sum_{n=1}^{N}\left(\rho_{n}(\mathbf{x})-\overline{\rho_{n}}(\mathbf{x})\right)^{2}}\right)$ is $8.75 \times 10^{-6}$, both of which are at least $10^{-5}$ times smaller than the mean. This demonstrates the accuracy of the coarse-graining approach.

\section{$7 \quad$ Divacancy binding energy}

As a second validation test and also to understand the propensity of vacancies to bind, we compute the divacancy binding energy in computational cells of sizes ranging from 54 to $2.5 \times 10^{6}$ atoms and compare our results with previous DFT calculations performed by Uesugi et al. [51]. As in the case of vacancies, the computational cell is terminated by enforcing bulk conditions on all fields at the boundary of the cell and the number of ESPs and atoms are selected such that the divacancy binding energies are numerically converged. In all our simulations we relax the atomic positions. Following the work of Uesugi et al. [51, the divacancy binding energy $E_{d b, i}$ is given as the difference between the formation energy of two vacancies $\left(2 E_{v f}\right)$ and the formation energy of a divacancy $\left(E_{d f, i}\right)$ :

$$
E_{d b, i}=2 E_{v f}-E_{d f, i}, \quad E_{d f, i}=M E_{\min }-\frac{M}{M-2} E(M-2)
$$

where the subindex $i$ refers to the different divacancy complex as shown in Figure 17. Figure 17a shows the spatial geometry of the six different divacancy complexes while Figure $17 \mathrm{~b}$

\footnotetext{
${ }^{2}$ We use the same Hamiltonian obtained using the coarse-grained $V_{\text {eff }}$ and the same Fermi level for both for consistent comparison.
} 
Table 4: Divacancy binding energy for Mg. Values predicted by MacroDFT and other DFT simulations. For MacroDFT, we detail the binding energy computed for two cell sizes. The number of atoms in the simulation cells is detailed between parenthesis. The values for comparison are taken from Ref. [51]. All binding energies are in eV.

\begin{tabular}{lccc}
\hline \hline & DFT(96) & MacroDFT (128) & MacroDFT (3456) \\
\hline$E_{2 V, 1 N N}$ & 0.06 & 0.076 & 0.106 \\
$E_{2 V, 2 N N}$ & 0.07 & 0.081 & 0.096 \\
$E_{2 V, 3 N N}$ & -0.01 & 0.024 & 0.057 \\
$E_{2 V, 4 N N}$ & 0.01 & 0.025 & 0.048 \\
$E_{2 V, 5 N N}$ & 0.01 & 0.023 & 0.038 \\
$E_{2 V, 6 N N}$ & 0.01 & 0.013 & 0.034 \\
\hline \hline
\end{tabular}

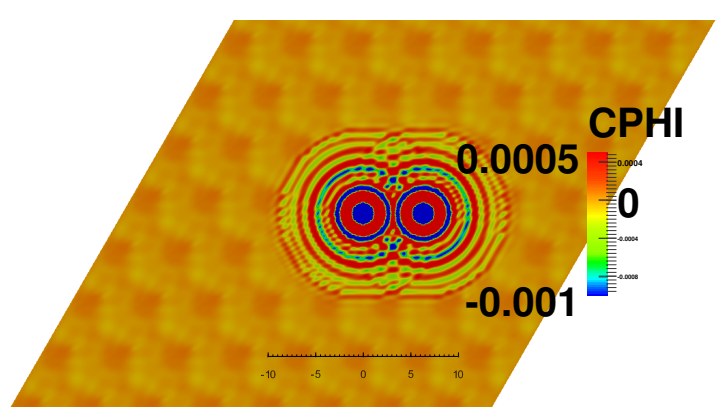

(a)

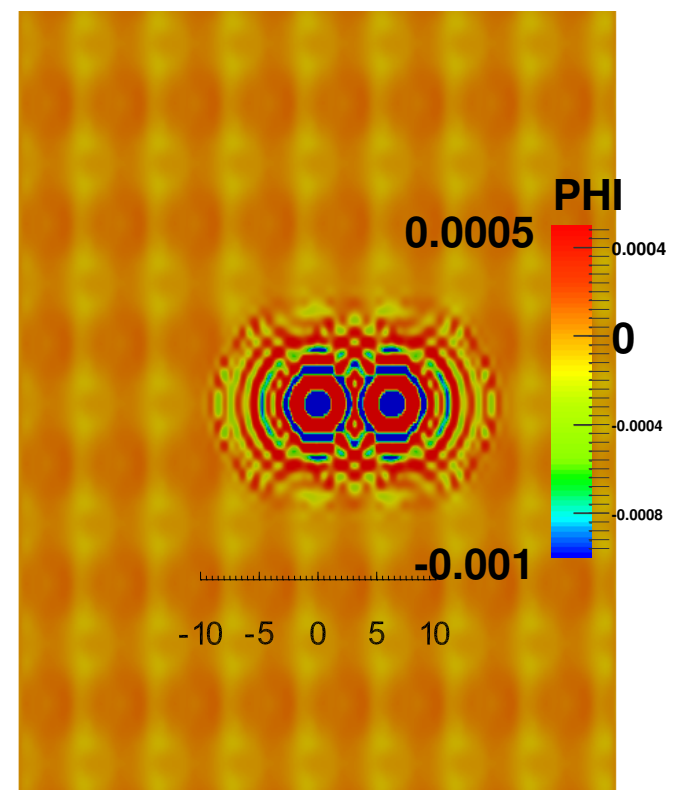

(c)

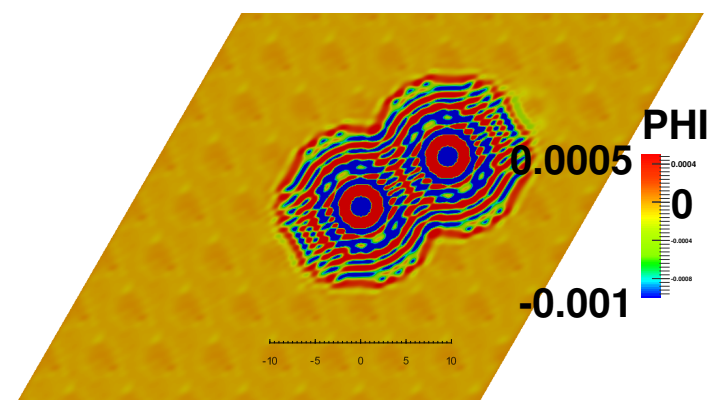

(b)

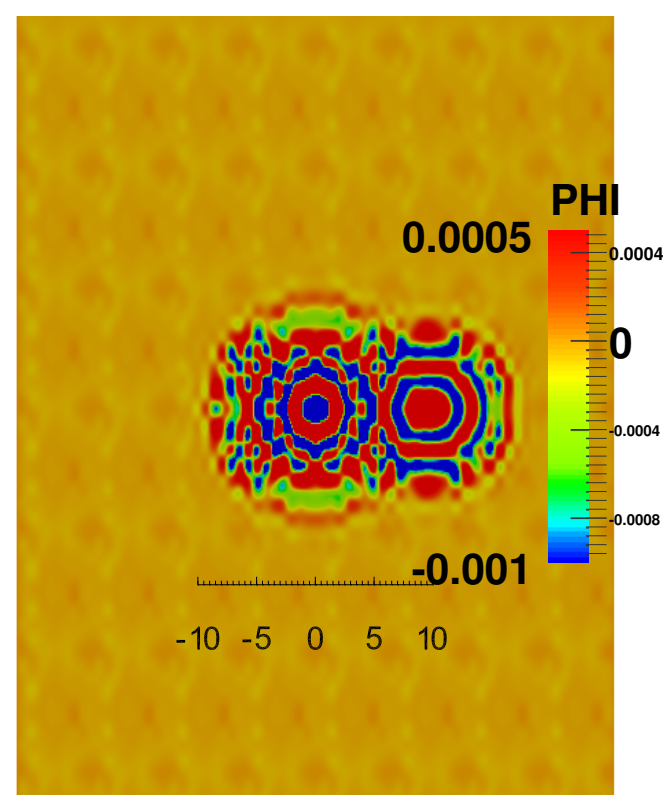

(d)

Figure 18: The corrector electrostatic potential (deviation from that of a perfect crystal) in a $\mathrm{Mg}$ $2.5 \times 10^{6}$-atom specimen with a divacancy. (a,c) Basal and prismatic planes of a $2 \mathrm{NN}$ divacancy. (b,d) basal and prismatic planes of a $5 \mathrm{NN}$ divacancy. The inset length-scale bar is in Bohr, and the potential in Hartree/electronic charge. 
shows the divacancy binding energy with the crystal size for these various configurations. Note that there is a very significant cell-size effect. The binding gets stronger for the larger cells for each configuration, achieving convergence approximately between 1,000 to 10,000 atoms. Also, note that the binding energies (when converged with cell size) orders with neighbor distance: it is the strongest for the nearest neighbor and weakest for the farthest neighbor.

Table 4 compares previous results obtained by Uesugi et al. [51] in Mg using plane wave code and Troullier-Martins non-local pseudopotential [37] with 96 atoms with our results for 128 atoms and 3456 atoms. Note that our calculations for the small cells are consistent with the previous results.

Figure 18 shows the corrector electrostatic field (i. e., the difference between the actual electrostatic field and that in a perfect crystal) on the basal and prismatic planes near the divacancy for a $2 \mathrm{NN}$ divacancy $(\mathrm{a}, \mathrm{c})$ and a $5 \mathrm{NN}$ divacancy $(\mathrm{b}, \mathrm{d})$. Comparing with Figure 12, we see that the oscillations near each vacancy is largely unchanged except for the overlap. So, the divacancy binding energy comes largely from the overlap. Since the overlap is larger closer the two vacancies, the binding energy orders with the distance between the nearest neighbors. Further, the computational cell has to be large enough to compute these oscillations, and this explains the slow convergence with respect to cell size.

Before we proceed, it is instructive to contrast the behavior of divacancies in $\mathrm{Mg}$ with those in Aluminum where divacancy complex is unstable for small computational cells [58, 51, 18. In $\mathrm{Al}$, there is a stronger directional bonds between near-neighbors in addition to the oscillations. The loss of the directional bond due to the divacancy causes an increase of energy while the overlap of the oscillations causes a decrease of energy. In small cells, the oscillations are not accurately accounted for and thus cause an overall increase in energy. Since the bonding is weak in $\mathrm{Mg}$, even small cells lead to a decrease of energy.

\section{Dependence of vacancy formation energy on volu- metric deformation}

As a final case study, we study how the vacancy formation energy changes with applied pressure. This is of particular interest for two reasons. First, it demonstrates the ability of MacroDFT under varying boundary conditions. Second, $\mathrm{Mg}$ alloys have been proposed for applications where they may be subjected to dynamic deformations where tension can lead to spall [59, 60]. The calculations use a $2.5 \times 10^{6}$ atoms specimen.

Due to the anisotropy of Mg-single crystals, we use the following procedure. We apply a boundary conditions corresponding to the deformation gradient

$$
\mathbf{F}=\left(\begin{array}{ccc}
\lambda_{1} & 0 & 0 \\
0 & \lambda_{1} & 0 \\
0 & 0 & \lambda_{2}
\end{array}\right)
$$

to the specimen, allow the atoms to relax and compute the energy. We repeat this for various ratios of $\lambda_{2} / \lambda_{1}$ for each fixed $\lambda_{1}^{2} \lambda_{2}$ to obtain the optimal value of this ratio. The formation energy as a function of the deformation gradient is defined as 


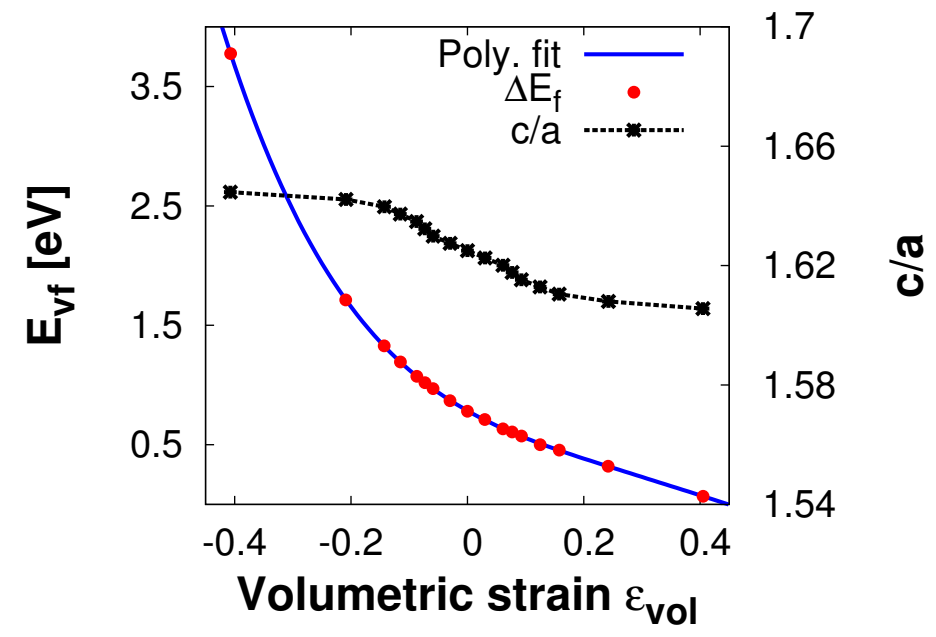

(a)

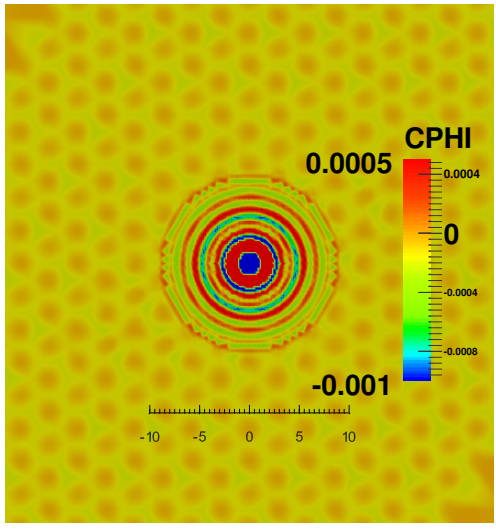

(b)

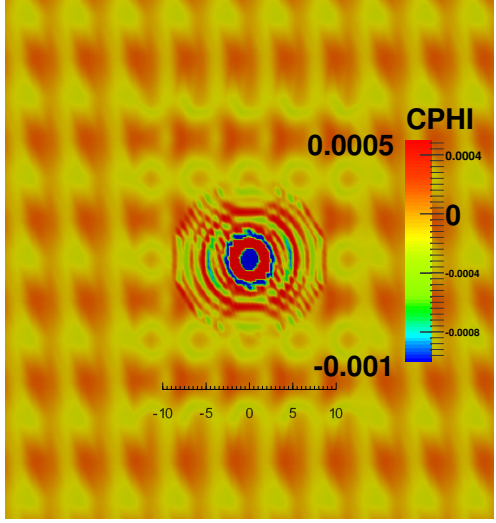

(d)

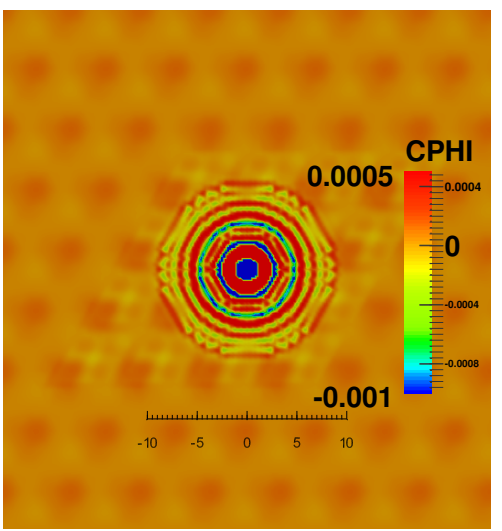

(c)

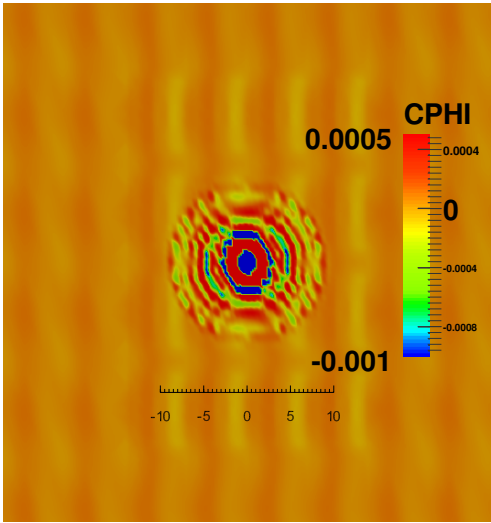

(e)

Figure 19: (a) The vacancy formation energy and the specimen c/a ratio for $\mathrm{Mg}$ as function of the volumetric deformation. (b,d) The corrector electrostatic potential on the basal and prismatic planes for 0.1 compression. (c,e) The corrector electrostatic potential on the basal and prismatic planes for 0.1 tension. The inset length-scale bar in (b-d) is in Bohr, and the potential in Hartree/electronic charge. 


$$
E_{v f}(M, \mathbf{F})=E(M, \mathbf{F})-\frac{M}{M-1} E(M-1, \mathbf{F})
$$

Figure 19(a) shows how the vacancy formation energy and the specimen $c / a\left(\lambda_{2} / \lambda_{1}\right)$ changes with volumetric deformation. The corrector electrostatic field for 0.1 compression is shown in Figure 19(b,d) while the corrector electrostatic field for 0.1 tension is shown shown in Figure 19(c,e). We see that the vacancy formation energy increases with compression. Comparing with Figure 12 we see that the oscillations near the vacancy change a little, but this is overcome by the large changes in the periodic fields. Consequently, $E_{\min }$ rises faster than $E(M-1)$ in 49 ). The vacancy formation energy decreases with tension but more gently, and this is consistent with the changes in the corrector electric field.

\section{Conclusions}

We have developed a sublinear scaling method for the study of crystal defects using $a b$ initio Density Functional Theory. The work exploits the properties of the Linear Scaling Spectral Gauss Quadrature (LSSGQ) [19] in a variable resolution numerical discretization or coarse-graining (CG) scheme. We have implemented the method in our computational platform MacroDFT. We have demonstrated the method and the implementation by studying point defects in HCP Magnesium. We have verified our approach and implementation by comparing our results with those obtained using established plane wave methods.

Our implementation at full resolution (no CG) displays linear scaling for up to 1024 atoms (Figure 8). Further a hybrid approach of MPI and OpenMP provides the optimal parallel performance achieving up to $80 \%$ speed-up with 4096 processors (Figure 9).

The CG improves on this and provides significant reduction of effort for especially large specimens. The current implementation of MacroDFT illustrates how by exploiting the decay properties of defects, one can accurately achieve sublinear scaling with number of electrons for crystalline solids and excellent performance on massively parallel supercomputers.

In our demonstration with vacancies, the method displays dramatically sublinear scaling (Table 2, Figure 15) and enables the computation of a billion atom specimen. This capability allows us to show that the vacancy formation energy converges slowly with cell size (Figure 11). This slow convergence, typical of crystal defects, is due to two reasons, first due to Friedel-type oscillations that extend to a radius of over 10 Bohr and second due to the slow decay of the elastic atomic displacements (Figure 12). Similar slow convergence with respect to computational cell size is also observed for divacancies (Figure 17). We have also studied the effect of pressure on a vacancy. Our study shows that under large hydrostatic tensile stress the vacancy formation energy goes to small values very fast. This finding is significant in relation to the analysis of dynamic failure, where the nucleation of point defects plays a critical role in the spall behavior of the material. 


\section{Acknowledgements}

Research was sponsored by the Army Research Laboratory and was accomplished under Cooperative Agreement Number W911NF-12-2-0022. The views and conclusions contained in this document are those of the authors and should not be interpreted as representing the official policies, either expressed or implied, of the Army Research Laboratory or the U.S. Government. The U.S. Government is authorized to reproduce and distribute reprints for Government purposes notwithstanding any copyright notation herein. KB and MO also acknowledge the partial financial support of the National Science Foundation through the PIRE grant No. OISE-0967140. This research used resources of the Argonne Leadership Computing Facility, which is a DOE Office of Science User Facility supported under Contract DE-AC02-06CH11357.

\section{Appendix: Force on a representative atom}

For ease of presentation, we assume that the underlying crystal lattice is a Bravais lattice (one atom per unit cell), but this assumption can easily be modified. We also recall our assumption that there are no solutes in the coarse-grained region, and assume that we have identical atoms everywhere. Under these assumptions, $b(\boldsymbol{x})=\sum_{a \in \mathcal{P}_{a}} b_{0}\left(\boldsymbol{x}-\boldsymbol{R}_{a}\right)$ for given $b_{0}$ that is obtained from the regularized nuclear charge (and possibly the local contribution of the pseudopotential) where the sum is taken over the set of all atoms $\mathcal{P}_{a}$. Note for future use that $b_{0}$ has very local support, i. e., it is zero outside a small region centered at the atomic position ${ }^{3}$. Using this representation for $b$, using the coarse-grained representation (37), and then using the divergence theorem (integrating by parts),

$$
\begin{aligned}
\boldsymbol{f}_{b} & =\int_{\Omega} \sum_{a \in \mathcal{P}_{a}}\left(\frac{\partial b_{0}}{\partial \boldsymbol{x}}\left(\boldsymbol{x}-\sum_{c \in \mathcal{P}_{\mathrm{QC}}} \Gamma_{a c} \boldsymbol{R}_{b}\right) \Gamma_{a b}\right) \phi(\boldsymbol{x}) d \boldsymbol{x} \\
& =-\int_{\Omega} \sum_{a \in \mathcal{P}_{a}}\left(b_{0}\left(\boldsymbol{x}-\sum_{c \in \mathcal{P}_{\mathrm{QC}}} \Gamma_{a c} \boldsymbol{R}_{b}\right) \Gamma_{a b}\right) \nabla \phi(\boldsymbol{x}) d \boldsymbol{x} .
\end{aligned}
$$

Note that this is the generalization of the Hellmann-Feynman force for the representative atom. Recall that $\Gamma_{a b}$ is zero for all atoms $a$ outside the atomistic elements which have representative atom $b$ as a node. Further, $b_{0}$ has very local support. Therefore, if the representative atom $b$ is in the region of very fine atomic region, the force can be evaluated with small effort.

This effort increases with the size of the atomistic element, but here we can exploit the

\footnotetext{
${ }^{3}$ Recall that $b_{0}$ is the regularized nuclear and possibly the local contribution of the pseudopotential and is zero outside a small region centered at the atomic position
} 
coarse-grained representation $(36)$ of the electrostatic field. Using this representation,

$$
\begin{aligned}
\boldsymbol{f}_{b}=- & \int_{\Omega} \sum_{a \in \mathcal{P}_{a}}\left(b_{0}\left(\boldsymbol{x}-\sum_{c \in \mathcal{P}_{\mathrm{QC}}} \Gamma_{a c} \boldsymbol{R}_{b}\right) \Gamma_{a b}\right) \nabla \phi^{0}(\boldsymbol{x}) d \boldsymbol{x} \\
& -\int_{\Omega} \sum_{a \in \mathcal{P}_{a}}\left(b_{0}\left(\boldsymbol{x}-\sum_{c \in \mathcal{P}_{\mathrm{QC}}} \Gamma_{a c} \boldsymbol{R}_{b}\right) \Gamma_{a b}\right) \nabla \phi^{c}(\boldsymbol{x}) d \boldsymbol{x} .
\end{aligned}
$$

Consider the term in (55).

$$
\begin{aligned}
\boldsymbol{f}_{b}^{1} & =-\int_{\Omega} \sum_{a \in \mathcal{P}_{a}}\left(b_{0}\left(\boldsymbol{x}-\sum_{c \in \mathcal{P}_{\mathrm{QC}}} \Gamma_{a c} \boldsymbol{R}_{b}\right) \Gamma_{a b}\right) \nabla \phi^{0}(\boldsymbol{x}) d \boldsymbol{x} \\
& =-\sum_{a \in \mathcal{P}_{a}} \Gamma_{a b} \int_{\Omega}\left(b_{0}\left(\boldsymbol{x}-\sum_{c \in \mathcal{P}_{\mathrm{QC}}} \Gamma_{a c} \boldsymbol{R}_{b}\right)\right) \nabla \phi^{0}(\boldsymbol{x}) d \boldsymbol{x}
\end{aligned}
$$

Recall that $b_{0}$ has very localized support and that $\nabla \phi^{0}$ is periodic in each atomistic element. Therefore,

$$
\int_{\Omega}\left(b_{0}\left(\boldsymbol{x}-\sum_{c \in \mathcal{P}_{\mathrm{QC}}} \Gamma_{a c} \boldsymbol{R}_{b}\right)\right) \nabla \phi^{0}(\boldsymbol{x}) d \boldsymbol{x}=\int_{\Omega_{0}^{e}} b_{0}(\boldsymbol{x}) \nabla \phi^{0}(\boldsymbol{x}) d \boldsymbol{x}
$$

where $\Omega_{0}^{e}$ is the crystalline unit cell in the eth element of the atomic mesh $\mathcal{T}_{\mathrm{QC}}$ that contains the atom $a$. It follows that

$$
\boldsymbol{f}_{b}^{1}=-\sum_{e \in \mathcal{T}_{\mathrm{QC}}^{b}} w_{e b} \int_{\Omega_{0}^{e}} b_{0}(\boldsymbol{x}) \nabla \phi^{0}(\boldsymbol{x}) d \boldsymbol{x}
$$

where $\mathcal{T}_{\mathrm{QC}}^{b}$ is the set of atomistic elements that contain representative atom $b$ as a node, $w_{e b}=\sum_{a \in\left(\mathcal{P}_{a} \cap \Omega_{e}\right)} \Gamma_{a b}$ where $\Omega_{e}$ is the eth element of the atomistic mesh. Note that the effort required for the evaluation of this term is independent of the number of atoms, i. e., $\mathcal{O}(1)$.

We now turn to the second term in (55). Recall that the corrector electrostatic field $\nabla \phi^{c}$ is constant in each element of the mesh $\mathcal{T}_{c}$ generated by the ESPs. It follows,

$$
\boldsymbol{f}_{b}^{2}=-\sum_{e \in \mathcal{T}_{\mathrm{QC}}^{b}} \sum_{f \in \mathcal{T}_{\mathrm{c}}^{e}} w_{e b}^{f} Z \nabla \phi_{f}^{c}
$$

where $\mathcal{T}_{\mathrm{c}}^{e}$ is the set of ESP elements that intersect with the atomistic element $e,-\nabla \phi_{f}^{c}$ is the corrector electric field in the ESP element $f, Z=\int_{\Omega} b_{0}(\boldsymbol{x}) d \boldsymbol{x}$,

$$
w_{e b}^{f}=\sum_{a \in\left(\mathcal{P}_{a} \cap \Omega_{e} \cap \omega_{f}\right)} \Gamma_{a b}
$$

where $\Omega_{e}$ is the eth element of the atomistic mesh and $\omega_{f}$ is the $f$ th element of the mesh generated by the ESPs. Again, the effort required for the evaluation of this term is independent of the number of atoms, i. e., $\mathcal{O}(1)$.

Putting these together and noting that $w_{e b}=\sum_{f \in \mathcal{T}_{c}^{e}} w_{e b}^{f}$, we obtain 48 . 


\section{References}

[1] P. Hohenberg and W. Kohn. Inhomogeneous electron gas. Physical Review, 136:B864B871, 1964.

[2] W. Kohn and L. J. Sham. Self-consistent equations including exchange and correlation effects. Physical Review, 140:A1133-A1138, 1965.

[3] J. R. Chelikowsky, N. Troullier, and Y. Saad. Finite-difference-pseudopotential method: Electronic structure calculations without a basis. Physical Review Letters, 72:1240$1243,1994$.

[4] G. Kresse and J. Furthmüller. Efficient iterative schemes for ab initio total-energy calculations using a plane-wave basis set. Physical Review B, 54:11169-11186, 1996.

[5] J. E. Pask, B. M. Klein, C. Y. Fong, and P. A. Sterne. Real-space local polynomial basis for solid-state electronic-structure calculations: A finite-element approach. Physical Review B, 59:12352-12358, 1999.

[6] S. Ismail-Beigi and T.A. Arias. New algebraic formulation of density functional calculation. Computer Physics Communications, 128:1-45, 2000.

[7] M. D. Segall, Philip J. D. Lindan, M. J. Probert, C. J. Pickard, P. J. Hasnip, S. J. Clark, and M. C. Payne. First-principles simulation: ideas, illustrations and the castep code. Journal of Physics: Condensed Matter, 14:2717-2744, 2002.

[8] E. Tsuchida. Ab initio molecular-dynamics study of liquid formamide. The Journal of Chemical Physics, 121:4740-4746, 2004.

[9] A. Castro, H. Appel, M. Oliveira, C. A. Rozzi, X. Andrade, F. Lorenzen, M. A. L. Marques, E. K. U. Gross, and A. Rubio. Octopus: a tool for the application of timedependent density functional theory. Physica Status Solidi (B), 243:2465-2488, 2006.

[10] G. Galli and M. Parrinello. Large scale electronic structure calculations. Physical

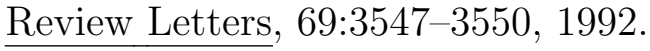

[11] F. Mauri, G. Galli, and R. Car. Orbital formulation for electronic-structure calculations with linear system-size scaling. Physical Review B, 47:9973-9976, 1993.

[12] S. Goedecker. Linear scaling electronic structure methods. Reviews of Modern Physics, 71:1085-1123, 1999.

[13] C.-K. Skylaris, P. D. Haynes, A. A. Mostofi, and M. C. Payne. Introducing onetep: Linear-scaling density functional simulations on parallel computers. The Journal of Chemical Physics, 122, 2005.

[14] M. Barrault, E. Cancès, W. W. Hager, and C. Le Bris. Multilevel domain decomposition for electronic structure calculations. Journal of Computational Physics, 222:86-109, 2007. 
[15] C. J. García-Cervera, J. Lu, Y. Xuan, and W. E. Linear-scaling subspace-iteration algorithm with optimally localized nonorthogonal wave functions for Kohn-Sham density functional theory. Physical Review B, 79:115110, 2009.

[16] D. R. Bowler and T. Miyazaki. $\mathcal{O}(n)$ methods in electronic structure calculations. Reports on Progress in Physics, 75:036503, 2012.

[17] E. Cancès, C. Le Bris, and P.-L. Lions. Molecular simulation and related topics: some open mathematical problems. Nonlinearity, 21:T165-T176, 2008.

[18] V. Gavini, K. Bhattacharya, and M. Ortiz. Quasi-continuum orbital-free densityfunctional theory: A route to multi-million atom non-periodic dft calculation. Journal of the Mechanics and Physics of Solids, 55:697-718, 2007.

[19] P. Suryanarayana, K. Bhattacharya, and M. Ortiz. Coarse-graining Kohn-Sham density functional theory. Journal of the Mechanics and Physics of Solids, 61:38-60, 2013.

[20] F. F. Abraham, J. Q. Broughton, N. Bernstein, and E. Kaxiras. Spanning the continuum to quantum length scales in a dynamic simulation of brittle fracture. Europhysics Letters, 44:783-787, 1998.

[21] C. Woodward. First-principles simulations of dislocation cores. Materials Science and Engineering: A, 400-401:59-67, 2005.

[22] J. R. Kermode, T. Albaret, D. Sherman, N. Bernstein, P. Gumbsch, M. C. Payne, G. Csányi, and A. De Vita. Low-speed fracture instabilities in a brittle crystal. Nature, 455:1224-1227, 2008.

[23] G. Lu, E. B. Tadmor, and E. Kaxiras. From electrons to finite elements: A concurrent multiscale approach for metals. Physical Review B, 73:024108, 2006.

[24] N. Bernstein, J. R. Kermode, and G. Csányi. Hybrid atomistic simulation methods for materials systems. Reports on Progress in Physics, 72:026501, 2009.

[25] X. Zhang, G. Lu, and W. A. Curtin. Multiscale quantum/atomistic coupling using constrained density functional theory. Physical Review B, 87:054113, 2013.

[26] M. Ponga. Multiscale modeling of point defects evolution at finite temperature: nanovoids and vacancies. Ph. D. Thesis, University of Seville, Spain, 2013.

[27] E. B. Tadmor, M. Ortiz, and R. Phillips. Quasicontinuum analysis of defects in solids. Philosophical Magazine, 73:1529-1563, 1996.

[28] M. A. Meyers and C. T. Aimone. Dynamic fracture (spalling) of metals. Progress in Materials Science, 28:1-96, 1983.

[29] M. Finnis. Interatomic Forces in Condensed Matter. Oxford Series on Materials Modelling 1. Oxford University Press, 2003. 
[30] R.G. Parr and W. Yang. Density-Functional Theory of Atoms and Molecules. International Series of Monographs on Chemistry. Oxford University Press, 1989.

[31] J. P. Perdew and Y. Wang. Accurate and simple analytic representation of the electrongas correlation energy. Phys. Rev. B, 45:13244-13249, Jun 1992.

[32] D. M. Ceperley and B. J. Alder. Ground state of the electron gas by a stochastic method. Phys. Rev. Lett., 45:566-569, Aug 1980.

[33] P. Suryanarayana, V. Gavini, T. Blesgen, K. Bhattacharya, and M. Ortiz. Non-periodic finite-element formulation of Kohn-Sham density functional theory. Journal of the Mechanics and Physics of Solids, 58:256-280, 2010.

[34] G. H. Golub and G. Meurant. Matrices, Moments and Quadrature with Applications. Princeton Series in Applied Mathematics, 2009.

[35] X. Wang, T. Blesgen, K. Bhattacharya, and M. Ortiz. A variational framework for spectral approximations of Kohn-Sham Density Functional Theory. Archive for Rational Mechanics and Analysis, in review, 2014.

[36] C. Huang and E. A. Carter. Transferable local pseudopotentials for magnesium, aluminum and silicon. Physical Chemistry Chemical Physics, 10:7109-7120, 2008.

[37] N. Troullier and J. L. Martins. Efficient pseudopotentials for plane-wave calculations. Phys. Rev. B, 43:1993-2006, 1991.

[38] L. Kleinman and D. M. Bylander. Efficacious Form for Model Pseudopotentials. Physical Review Letters, 48:1425-1428, 1982.

[39] T.J.R. Hughes. The finite element method. Dover Publications, 2000.

[40] J. Knap and M. Ortiz. An analysis of the quasicontinuum method. Journal of the Mechanics and Physics of Solids, 49:1899-1923, 2001.

[41] B. Fornberg. A Practical Guide to Pseudospectral Methods. Cambridge Monographs on Applied and Computational Mathematics. Cambridge University Press, 1998.

[42] Y. Saad and M. Schultz. Gmres: A generalized minimal residual algorithm for solving nonsymmetric linear systems. SIAM Journal on Scientific and Statistical Computing, 7:856-869, 1986.

[43] S. Balay, J. Brown, K. Buschelman, W. D. Gropp, D. Kaushik, M. G. Knepley, L. C. McInnes, B. F. Smith, and H. Zhang. PETSc Web page, 2011. www.mcs.anl.gov/petsc.

[44] H. Fang and Y. Saad. Two classes of multisecant methods for nonlinear acceleration. Numerical Linear Algebra with Applications, 16:197-221, 2009.

[45] W. T. Vetterling, W. H. Press, B. P. Flannery, and S. A. Teukolsky. Numerical Recipes Example Book C++. Cambridge University Press, 2002. 
[46] G. P. M. Leyson, W. A. Curtin, L. G. Hector, and C. F. Woodward. Quantitative prediction of solute strengthening in aluminium alloys. Nature Materials, 9:750-755, 2010.

[47] C. Woodward, D. R. Trinkle, L. G. Hector, and D. L. Olmsted. Prediction of dislocation cores in aluminum from density functional theory. Physical Review Letters, 100:045507, 2008.

[48] F. D. Murnaghan. The compressibility of media under extreme pressures. Proceedings of the National Academy of Sciences, 30:244-247, 1944.

[49] N. Chetty, M. Weinert, T. S. Rahman, and J. W. Davenport. Vacancies and impurities in aluminum and magnesium. Physical Review B, 52:6313-6326, 1995.

[50] H. Krimmel and M. Fähnle. Ab initio calculation of the formation and migration energies for monovacancies in mg. Physical Review B, 62:5489-5491, 2000.

[51] T. Uesugi, M. Kohyama, and K. Higashi. Ab initio on divacancy binding energies in aluminum and magnesium. Physical Review B, 68:184103, 2003.

[52] C. Mairy, J. Hillairet, and D. Schumacher. Energie de formation et concentration d'équilibre des lacunes dans le magnésium. Acta Metallurgica, 15:1258-1261, 1967.

[53] C. Janot, D. Malléjac, and B. George. Vacancy-formation energy and entropy in magnesium single crystals. Physical Review B, 2:3088-3098, 1970.

[54] P. Tzanetakis, J. Hillairet, and G. Revel. The formation energy of vacancies in aluminium and magnesium. Physica Status Solidi (B), 75:433-439, 1976.

[55] A. Vehanen and K. Rytsölä. Proceedings of the international school of physics. North-Holland, Utrecht, 1981.

[56] H. Si. TetGen Web page, 2011. http://wias-berlin.de/software/tetgen/.

[57] W. Kohn. Density functional and density matrix method scaling linearly with the number of atoms. Physical Review Letters, 76:3168-3171, 1996.

[58] K. M. Carling, G. Wahnström, T. R. Mattsson, N. Sandberg, and G. Grimvall. Vacancy concentration in al from combined first-principles and model potential calculations. Physical Review B, 67:054101, 2003.

[59] P.J. Hazell, G.J. Appleby-Thomas, E. Wielewski, C. Stennett, and C. Siviour. The influence of microstructure on the shock and spall behaviour of the magnesium alloy, elektron 675. Acta Materialia, 60:6042-6050, 2012.

[60] K. Eswar Prasad, B. Li, N. Dixit, M. Shaffer, S.N. Mathaudhu, and K.T. Ramesh. The dynamic flow and failure behavior of magnesium and magnesium alloys. The Journal of The Minerals, Metals \& Materials Society (TMS), 66:291-304, 2014. 